\title{
Evaluation of Staphylococcus aureus Lipoproteins: Role in Nutritional Acquisition and Pathogenicity
}

\author{
Shideh V. Shahmirzadi ${ }^{\dagger}$, Minh-Thu Nguyen ${ }^{\dagger}$ and Friedrich Götz * \\ Microbial Genetics, Interfaculty Institute of Microbiology and Infection Medicine, University of Tübingen, Tübingen, Germany
}

\section{OPEN ACCESS}

Edited by:

Marie-Pierre Chapot-Chartier

Centre de Recherches de

Jouy-en-Josas (INRA), France

Reviewed by:

lain Sutcliffe,

Northumbria University, UK

Alain Rincé,

Normandie-Université, France

*Correspondence:

Friedrich Götz

friedrich.goetz@uni-tuebingen.de

${ }^{\dagger}$ These authors have contributed equally to this work.

Specialty section

This article was submitted to Infectious Diseases,

a section of the journal

Frontiers in Microbiology

Received: 28 June 2016

Accepted: 24 August 2016

Published: 13 September 2016

Citation:

Shahmirzadi SV, Nguyen M-T and

Götz F (2016) Evaluation of

Staphylococcus aureus Lipoproteins:

Role in Nutritional Acquisition and

Pathogenicity.

Front. Microbiol. 7:1404.

doi: 10.3389/fmicb.2016.01404
Bacterial lipoproteins (Lpp) represent a major class of membrane proteins. They are distinguished by a lipid moiety at the $\mathrm{N}$-terminus by which they are anchored either in the outer leaflet of the cytoplasmic membrane or, in Gram-negative bacteria, also in the inner leaflet of the outer membrane. In Gram-positive bacteria Lpp significantly contribute to nutrient transport, Toll-like receptor 2 activation and pathogenicity. Here we examine the Lpp of Staphylococcus aureus USA300, as a prototype for a multiple antibiotic resistant and community-acquired pathogen that is rapidly spreading worldwide. The compiled Lpp were grouped according to the postulated function and dissemination of homologs in the genus Staphylococcus and beyond. Based on this evaluation we also point out Lpp as promising vaccine candidates.

Keywords: Staphylococcus, S. aureus USA300, lipoprotein, lipoprotein functions, lipoprotein dissemination, ion transporters, pathogenicity

\section{INTRODUCTION}

Bacterial lipoproteins (Lpp) are a distinctive class of membrane-anchored proteins. They contain a N-terminal lipid modification, the $N$-acyl-S-diacyl-glyceryl-cysteine (Hantke and Braun, 1973). There are three cytoplasmic membrane localized enzymes involved in the biogenesis of Lpp: the phosphatidylglycerol-prolipoprotein diacylglyceryl transferase (Lgt) (Sankaran and Wu, 1994), the specific signal peptidase II (Lsp) that recognizes the diacylglyceryl modification and cleaves between the amino acid at position -1 and the lipid-modified cysteine residue (Hussain et al., 1982), and finally the $N$-acyltransferase (Lnt) to form $N$-acyl diacylglyceryl cysteine (Gan et al., 1995). This maturation of Lpp is uniform in Gram-negative and-positive bacteria. In Gram-positive bacteria Lpp are anchored in the outer leaflet of the cytoplasmic membrane and may extend into the cell wall and beyond. Although their anchoring at the cell envelope is completely different from the covalently cell wall anchored proteins mediated by the sortase (Mazmanian et al., 2001), there is an overlap which have both in common, namely, the binding to external components, such as nutrients and host proteins. Particularly this function is affected when the maturation of pre-Lpp by the Lgt and Lsp does not take place.

The crucial role of maturation of pre-Lpp by the Lgt and Lsp for virulence and TLR2 signaling has been reviewed recently (Nguyen and Götz, 2016). For example the $\Delta l g t$ mutants of various S. aureus strains were severely affected in immune stimulation and pathogenicity (Stoll et al., 2005; Schmaler et al., 2009); and the $\Delta l g t$ mutants were also affected in iron acquisition under infectious conditions (Schmaler et al., 2009, 2010). In many other Gram-positive bacteria like Mycobacterium tuberculosis (Sander et al., 2004), Streptococcus pneumoniae (Petit et al., 2001; Khandavilli et al., 2008), Streptococcus agalactiae (Henneke et al., 2008; Bray et al., 2009), Streptococcus pyogenes, 
and Streptococcus equi (Sutcliffe and Harrington, 2002; Hamilton et al., 2006; Weston et al., 2009; Sutcliffe et al., 2012) or Listeria monocytogenes (Baumgärtner et al., 2007; Machata et al., 2008) deletion of the lgt or the lsp gene likewise impaired growth and pathogenicity.

By screening the $S$. aureus N315 genome with the new Lpp search program ParSeq (Schmollinger et al., 2004), more than 70 putative Lpp were identified, but only 55 contained a signal peptide with the right length (Stoll et al., 2005). 35 of the Lpp could be annotated as transporters for iron, zinc, amino acid, oligopeptide, glycine betaine, sugar, and teichoic acid, other had enzymatic functions such heme/copper-type cytochrome/quinol oxidase, protein-disulfide isomerase, peptidyl-prolyl cis/trans isomerase (PrsA), or pyruvate-format-lyase-activating enzyme. In this report it has also been shown that SitC was one of the most abundant Lpp and that in a lgt mutant only $20-25 \%$ retained in the membrane, while the majority was released into the supernatant (Stoll et al., 2005). In the very detailed review by Sibbald et al. 43 core and a similar amount of variant Lpp were analyzed and they also mention that the translocation pathway is mostly Sec- but in some cases also Tat-mediated (Sibbald et al., 2006).

In the meantime the knowledge as to the function of Lpp in $S$. aureus has increased, justifying a reevaluation of the data. We carefully analyzed the Lpp in strain USA300 as an epidemic prototype and traced the distribution of each Lpp homolog in the $S$. aureus species, the Staphylococcus genus and beyond. This allows us to unravel housekeeping and virulence associated Lpp. It turned out that particularly pathogenic strains have a number of additional Lpp serving as transporters for nutrients and contributing to virulence and fitness.

\section{MATERIALS AND METHODS}

\section{Bioinformatic Study}

We collected all the predicted Lpp generated by different programs, for example Hidden Markov Model (Bagos et al., 2008), LipoP (Juncker et al., 2003), G+LPP (Sutcliffe and Harrington, 2002), G+LPPv2 (Rahman et al., 2008), LIPPREP (Taylor et al., 2006), Dolop (Babu et al., 2006), and Von Heijne (von Heijne, 1989). These lipoproteins were screened carefully by combination of the different values including hydrophobic plot (http://gcat.davidson.edu/DGPB/kd/kyte-doolittle.htm), lipobox sequence (http://www.mrc-lmb.cam.ac.uk/genomes/dolop/ lipobox.shtml) and the cleaving site by PREP-LIPO (http:// bioinformatics.biol.uoa.gr/PRED-LIPO/) or LipoP (http://www. cbs.dtu.dk/services/LipoP/). All Lpp sequences were blasted with other staphylococcal species and other genera by following programs NCBI protein blast (http://blast.ncbi.nlm.nih.gov/ Blast.cgi?PAGE=Proteins) or KEGG (http://www.genome.jp/ $\mathrm{kegg} / \mathrm{ko.html}$ ).

Abbreviations: Lpp, Lipoproteins; lgt, diacylglyceryl transferase enzyme encoding gene; Lpl, lipoprotein-like; S, Staphylococcus; TLR, Toll-like receptor; CC, clonal complex; AA, Amino acid; Numbers behind Lpp in parenthesis refer to the numbers in Table 1.

\section{Results and Discussion Evaluation of Lipoproteins (Lpp) in Staphylococcus aureus USA300}

Here, we re-evaluated the Lpp of a pathogenic S. aureus strain and categorized the Lpp according to the function and dissemination. Methicillin-resistant S. aureus USA300 was chosen as a model strain as it is a major source of communityacquired infections almost worldwide (Diep et al., 2006). The common Lpp prediction programs yielded different numbers of proposed Lpp in the 2560 genes of USA300 (http://biophysics.biol.uoa.gr/PRED-LIPO-results/): DOLOP (52), G+LPP (54), von heijne (66), PS51257 (68), PS00013 (65) and LipoP (68) and PRED-LIPO (63). Because of this heterogeneity each proposed Lpp was examined for the presence of an unambiguous Lpp signal peptide (LSP), which must fulfill three criteria: length between 16 and 40 amino acids, presence of a hydrophobic domain, followed by the lipobox. Based on these criteria we propose $67 \mathrm{Lpp}$ in USA300, which is $2.57 \%$ of all genes (Table 1). Three of the identified Lpp in USA300 (no 5,65 , and 64 of Table 1) were incorrectly annotated by using the wrong start codon; these Lpp were also recently detected in the supernatant of $S$. aureus Newman by proteomic analysis (Vu et al., 2016). The size of the Lpp ranged from 6 to $89 \mathrm{kDa}$, however, the average size was between 30 and $50 \mathrm{kDa}$.

\section{Functional Categorization of Lpp}

We grouped the Lpp according to their function. Most of the functions were deduced from studies of homologous counterparts in other bacteria, but in 12 cases the ascribed functions were confirmed by molecular/biochemical studies in $S$. aureus.

\section{Iron transporters}

The first group in Table 1 represent Lpp that are involved in iron acquisition which is extremely important in order for pathogenic bacteria to obtain some of the limited free iron ions during infection. For this reason intricate iron transport and iron regulatory systems have evolved in pathogenic bacteria to guarantee sufficient iron supply (Braun, 2001). It is therefore not surprising that $8 \mathrm{Lpp}$ are involved iron acquisition or utilization of host-derived heme iron (isd operon) as an iron source. Under iron limitation, iron(III)-hydroxamate siderophores are excreted as iron chelators by the FhuCBG system (Sebulsky et al., 2000) and the iron-loaded chelators are bound by two Lpp (FhuD1 and FhuD2) acting as receptors and representing the first step in iron acquisition. Although both receptors are homologous (41\% identity) they exhibit different activities. FhuD2 is conserved in other species and binds a broad spectrum of Fe chelators, such as ferric hydroxamate, and various siderophores, such as ferrichrome, ferrioxamineB, aerobactin, and coporgen. FhuD1, on the other hand, is only found in staphylococcal species and binds only ferrichrome and ferrioxamine $\mathrm{B}$, and exhibits a lower affinity for hydroxamate siderophore binding than FhuD2 (Sebulsky and Heinrichs, 2001; Sebulsky et al., 2004). The Lpp SirA belongs to the iron regulated SirABC operon (Heinrichs et al., 1999). Mutants of either sirA or sirB are unable to take up iron complexes, such as ferric hydroxamates, 
TABLE 1 | Lpp of S. aureus USA300.

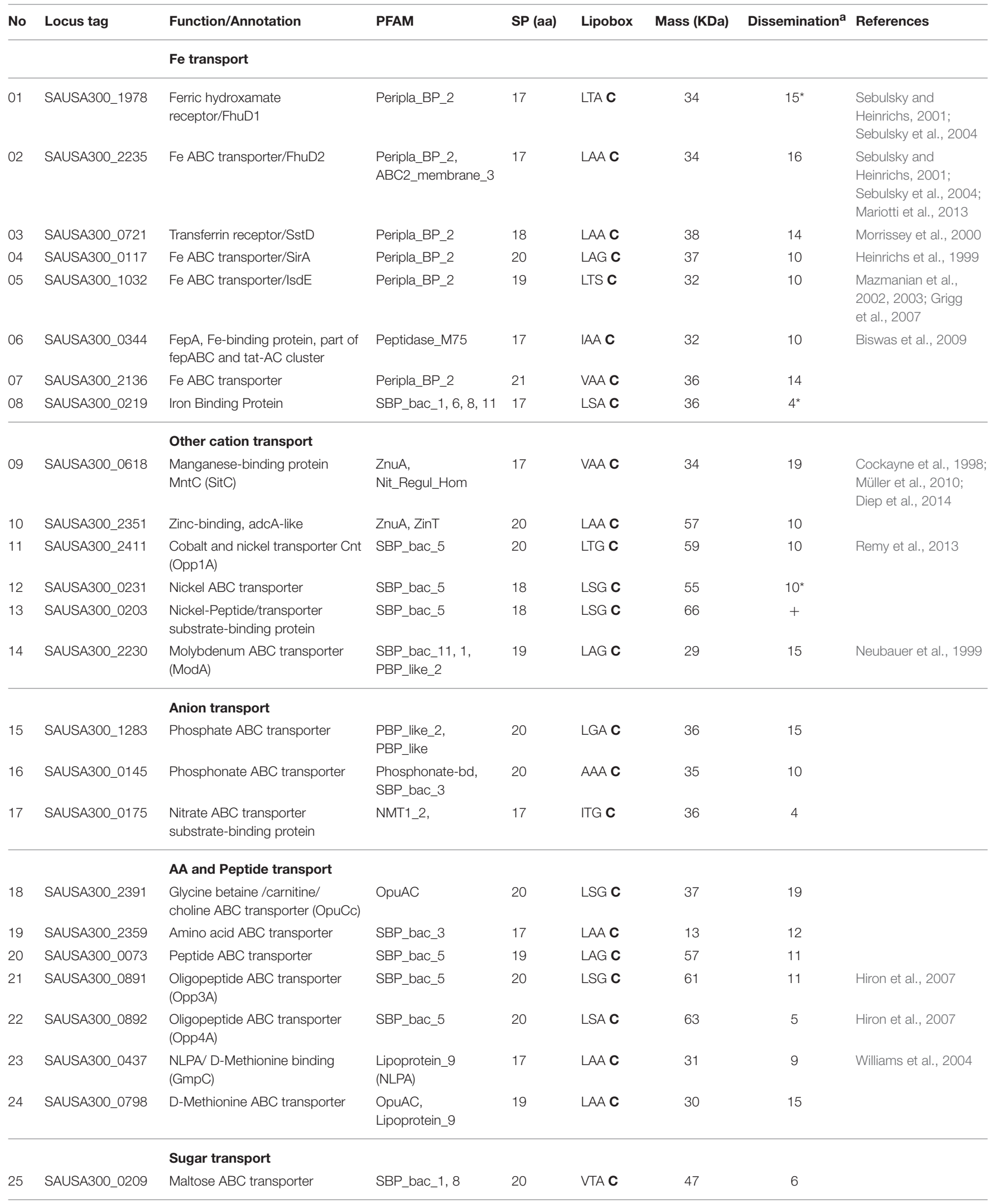

Miscellaneous functions 
TABLE 1 | Continued

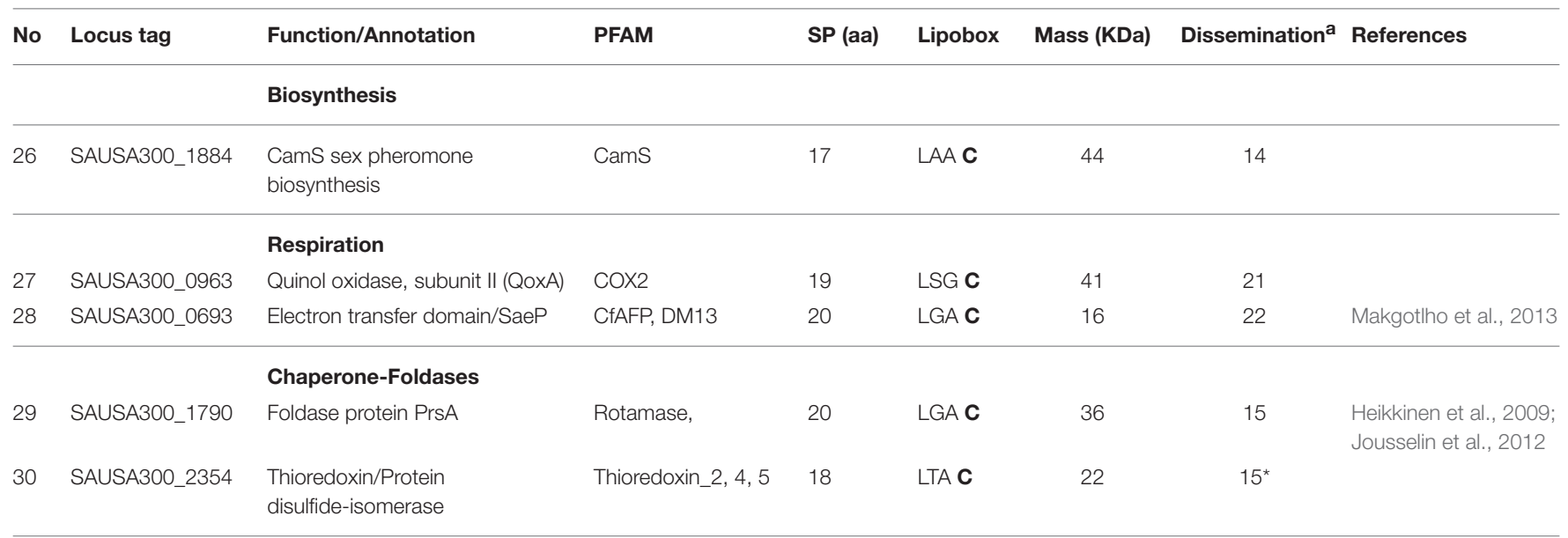

\begin{tabular}{|c|c|c|c|c|c|c|c|c|}
\hline \multirow[b]{2}{*}{31} & \multirow[b]{2}{*}{ SAUSA300_2046 } & \multicolumn{7}{|l|}{ Protein translocation } \\
\hline & & YidC (OxaA)-essential protein & OATP, 60KD_IMP & 19 & LAG C & 32 & 25 & \\
\hline & & $\begin{array}{l}\text { Phage and plasmid encoded } \\
\text { Lpp }\end{array}$ & & & & & & \\
\hline 32 & SAUSA300_1436 & PhiSLT ORF144-like & $\begin{array}{l}\text { DUF1510, Zip, } \\
\text { Presenilin }\end{array}$ & 17 & LTA C & 16 & $2^{*}$ & \\
\hline \multirow[t]{2}{*}{33} & $\begin{array}{l}\text { PUSA300 } \\
\text { HOUMR0011 }\end{array}$ & $\begin{array}{l}\text { Membrane bound penicillinase } \\
\text { BlaZ }\end{array}$ & & 16 & LSA C & 31 & 11 & $\begin{array}{l}\text { Nielsen and Lampen, } \\
\text { 1982b }\end{array}$ \\
\hline & & Lpl cluster & & & & & & \\
\hline 34 & SAUSA300_0410 & Lpl-1 vSa $\alpha$ specific & DUF576 & 32 & IAG C & 30 & + & Nguyen et al., 2015b \\
\hline 35 & SAUSA300_0411 & Lpl-2 vSa $\alpha$ specific & DUF576 & 22 & $\| G \mathbf{C}$ & 30 & + & Nguyen et al., 2015b \\
\hline 36 & SAUSA300_0413 & Lpl-3 vSa $\alpha$ specific & DUF576 & 23 & $\| G C$ & 30 & + & Nguyen et al., 2015b \\
\hline 37 & SAUSA300_0414 & Lpl-4 vSa $\alpha$ specific & DUF576 & 22 & VTS C & 28 & + & Nguyen et al., 2015b \\
\hline 38 & SAUSA300_0415 & Lpl-5 vSa $\alpha$ specific & DUF576 & 22 & IMG C & 29 & + & Nguyen et al., 2015b \\
\hline 39 & SAUSA300_0416 & Lpl-6 vSa $\alpha$ specific & DUF576 & 20 & MAG C & 29 & + & Nguyen et al., 2015b \\
\hline 40 & SAUSA300_0417 & Lpl-7 vSa $\alpha$ specific & DUF576 & 23 & IVG C & 30 & + & Nguyen et al., 2015b \\
\hline 41 & SAUSA300_0418 & Lpl-8 vSa $\alpha$ specific & DUF576 & 22 & VTS C & 29 & + & Nguyen et al., 2015b \\
\hline 42 & SAUSA300_0419 & Lpl-9 vSa $\alpha$ specific & DUF576 & 22 & IGG C & 30 & + & Nguyen et al., 2015b \\
\hline 43 & SAUSA300_2429 & Tandem Ipp & DUF576 & 22 & IGG C & 16 & $3^{*}$ & \\
\hline 44 & SAUSA300_2430 & Tandem Ipp & DUF576 & 23 & IGA C & 29 & + & \\
\hline 45 & SAUSA300_0100 & $\begin{array}{l}\text { Tandem Ipp/Conserved } \\
\text { staphylococcal antigen } 1 \mathrm{~A} \\
\text { (Csa1A) }\end{array}$ & DUF576 & 24 & TAG $\mathbf{C}$ & 28 & + & Schluepen et al., 2013 \\
\hline 46 & SAUSA300_0101 & Tandem Ipp & DUF576 & 24 & TAG C & 28 & + & \\
\hline 47 & SAUSA300_0102 & Tandem Ipp & DUF576 & 24 & TAG $\mathbf{C}$ & 28 & + & \\
\hline \multirow[t]{2}{*}{48} & SAUSA300_0103 & Tandem Ipp & DUF576 & 23 & TAG C & 28 & + & \\
\hline & & Unknown function & & & & & & \\
\hline 49 & SAUSA300_0079 & Unknown function & DUF1541 & 18 & LSA C & 20 & 17 & \\
\hline 50 & SAUSA300_0372 & Unknown function & PepSY & 18 & LTA C & 21 & $17^{\star}$ & \\
\hline 51 & SAUSA300_0377 & Unknown function & DUF1748 & 19 & LTG C & 23 & 14 & \\
\hline 52 & SAUSA300_1492 & Unknown function & & 16 & LAG C & 13 & $15^{\star}$ & \\
\hline 53 & SAUSA300_0992 & Cell-wall binding lipoprotein & YkyA, EzrA & 19 & LAG C & 23 & $13^{*}$ & \\
\hline 54 & SAUSA300_2403 & Unknown function & DUF1307 & 20 & LSA C & 17 & $12^{*}$ & \\
\hline 55 & SAUSA300_0724 & Unknown function & IncA, TarH & 19 & ISA C & 32 & 12 & \\
\hline 56 & SAUSA300_2315 & Unknown function & PA26, IncA, CLN3 & 17 & LAA C & 23 & $11^{*}$ & \\
\hline 57 & SAUSA300_2614 & Unknown function & DUF_1980 & 20 & LYS C & 42 & 6 & \\
\hline 58 & SAUSA300_0663 & Unknown function & PA26, IncA & 17 & LTG C & 15 & 5 & \\
\hline
\end{tabular}




\section{TABLE 1 | Continued}

\begin{tabular}{llllllll}
\hline No & Locus tag & Function/Annotation & PFAM & SP (aa) & Lipobox & Mass (KDa) & Dissemination $^{\mathbf{a}}$ References \\
\hline 59 & SAUSA300_1106 & Unknown function & FAM176 & 18 & VAG C & 35 & $5^{\star}$ \\
60 & SAUSA300_0303 & Unknown function & DUF4467 & 17 & LAG C & 14 & $5^{\star}$ \\
61 & SAUSA300_1478 & Unknown function & DUF4467 & 17 & LSA C & 13 & $3^{\star}$ \\
62 & SAUSA300_1376 & Unknown function & DUF1672 & 17 & LSG C & 34 & $2^{\star}$ \\
63 & SAUSA300_1379 & Unknown function & DUF1672 & 17 & LSG C & 34 & $2^{\star}$ \\
64 & SAUSA300_1440 & Unknown function & DUF1672 & 17 & LGG C & 34 & $2^{\star}$ \\
65 & SAUSA300_1742 & Unknown function & & 18 & LTA C & 23 & $2^{\star}$ \\
66 & SAUSA300_1741 & Unknown function & ETRAMP, & 18 & LTA C & 6 & + \\
67 & SAUSA300_0769 & Unknown function & MycO_19_kDa & & & & +
\end{tabular}

${ }^{a}$ The number indicates the number of staphylococcal species in which the corresponding homologues gene/protein (more than $40 \%$ identity over the whole protein length) is present. + S. aureus specific gene.

*Staphylococcal specific gene.

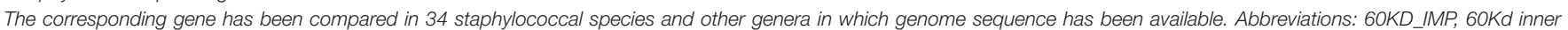

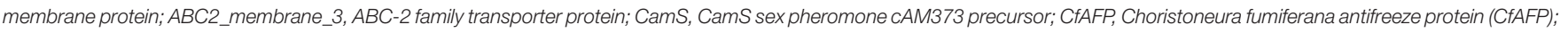

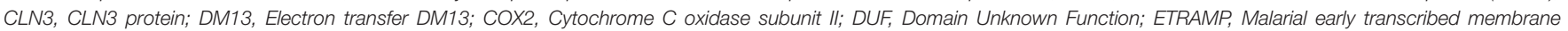

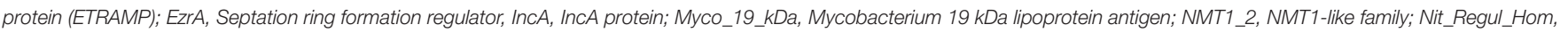

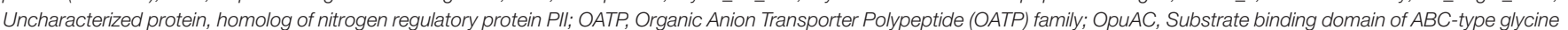

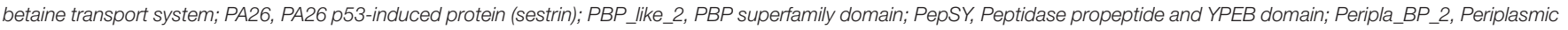

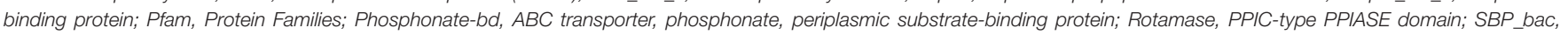

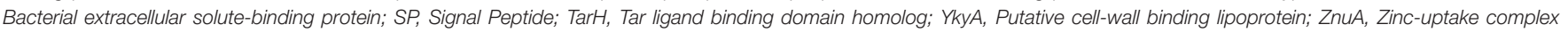
component A periplasmic; ZinT, (YodA) periplasmic lipocalin-like zinc-recruitment; Zip, ZIP Zinc transporter.

ferric enterobactin or ferric citrate but they retain the ability to produce staphylobactin (Dale et al., 2004). During infection and iron limitation $S$. aureus is also able to use heme as an iron source via the complex isdCDEFsrtBisdG operon. IsdA and IsdB are involved in heme uptake, which is derived from the host hemoglobin. IsdC is a cell wall localized transporter, while IsdD, IsdE, and IsdF are the membrane translocation factors, and IsdG, is the cytoplasmic heme-iron binding protein (Mazmanian et al., 2003). While IsdA, IsdB, and IsdC are covalently cellwall bound by sortase anchoring, IsdE is the heme-binding Lpp (Mazmanian et al., 2002; Grigg et al., 2007). Another ironlimited expressed siderophore transport system is composed by 4 proteins SstA,B,C, and D where SstD is the Lpp (Morrissey et al., 2000). The twin-arginine translocation (Tat) pathway, is present in only some staphylococcal species, and is composed of TatA and TatC (Biswas et al., 2009). The tat AC operon is associated with the fepABC operon. FepA is a Lpp and mediates iron binding, FepB with its typical TAT signal peptide is the iron-dependent peroxidase (FepB), and FepC is supposed to be the high affinity iron permease (Biswas et al., 2009). The advantage of the fep-tat cluster could lie in iron uptake and in the external detoxification of reactive oxygens. Indeed, the iron uptake under iron limitation was significantly decreased in the fep-tat mutants, which were less virulent in a mouse kidney abscess model (Biswas et al., 2009). The last two Lpp in this group (7 and 8) were annotated as iron binding Lpp, however, their function is unknown. The USA300_2136 (7) is encoded in the operon with two other genes, both were annotated as ion $\mathrm{ABC}$ transporter permease proteins containing the same motifs as FecCD and ABC3. The USA300_0219 (8) contains a SBP_bac motif (Table 1).

\section{Other cation and anion transporters}

Beside iron transporters there are other cation transporters for $\mathrm{Co}, \mathrm{Cu}, \mathrm{Mn}, \mathrm{Mo}, \mathrm{Ni}, \mathrm{Zn}$ ions, which become important when these trace elements are limited. In some cases, they are crucial in infection. One of the most abundant Lpp in S. aureus is the 33 $\mathrm{kDa} \mathrm{MntC}$ (SitC) (Stoll et al., 2005). Originally it was referred to as SitC because its protein sequence shares $77 \%$ identity to SitC of $S$. epidermidis, where it has been described as being involved in iron transport (Cockayne et al., 1998). However, instead of being involved in iron transport, $\mathrm{SitC}$ has recently been found to have a role in manganese $(\mathrm{Mn})$ transport and is part of the MntABC system (Horsburgh et al., 2002; Diep et al., 2014). To avoid confusion we use here the term MntC (SitC). Three Lpp were annotated as Ni transporters. The Opp1 transport system is involved in cobalt and nickel uptake and has been renamed as Cnt (Remy et al., 2013). There is a Cnt related protein (25\% identity) encoded by SAUSA300_0203 that was annotated as peptide/nickel transport system. The Lpp, ModA, is part of the molybdate transporter complex (ModABC) (Neubauer et al., 1999).

Based on annotation the following Lpp are involved in phosphate (USA300_1283), phosphonate (USA300_0145) and nitrate transport (USA300_0175). They are encoded with other genes in the same operon involved in the transport apparatus.

\section{Amino acid and peptide transporters}

There are 7 Lpp involved in amino acid and peptide transport. Opp3 is a main system that provides oligopeptides as a nutritional source (Hiron et al., 2007), and GmpC binds the dipeptide glycylmethionine and is part of an $\mathrm{ABC}$ transporter system (Williams et al., 2004). 


\section{Sugar transport}

There is only one Lpp involved in sugar transport, the maltose ABC transporter.

\section{Lpp with miscellaneous functions}

The group of miscellaneous Lpp comprise Lpp with diverse functions. They are involved in sex pheromone biosynthesis, in terminal electron transfer to oxygen (QoxA) or in global regulation (SaeP). QoxA is part of the terminal cytochrome aa3 quinol oxidase encoded by qoxABCD (Götz and Mayer, 2013; Hammer et al., 2013). The protein complex SaePQ activates the phosphatase activity of sensor kinase SaeS in the SaeRS two-component system of S. aureus (Jeong et al., 2012). The Sae system controls the expression of numerous virulence factors, such as the extracellular adherence protein (Eap), which facilitates host cell invasion (Makgotlho et al., 2013). The chaperon, PrsA, belongs to the parvulin PPIase family (pepdidylprolyl cis/trans isomerase) that assists posttranslocational folding at the outer surface of the cytoplasmic membrane (Heikkinen et al., 2009). In Bacillus, PrsA is an essential protein. In S. aureus it is not essential, but a prsA deletion mutant is impaired in post-transcriptional maturation of $\mathrm{PBP} 2 \mathrm{~A}$ and shows therefore decreased methicillin resistance (Jousselin et al., 2012, 2015). There is another chaperone annotated as a thioredoxin/protein disulfide-isomerase related to DsbA that catalyzes disulfide formation and isomerization and that acts simultaneously as a chaperone by preventing protein aggregation (Kouwen et al., 2007). YidC (short form of OxaI-like protein) acts as a membrane integrase for Sec-dependent substrates, such as ATP synthase subunit a (Foa) or cytochrome $b_{3}$ oxidase CyoA subunit, but can also act as a chaperone and as an assembly site for membrane protein folding (Wang and Dalbey, 2011). YidC is highly conserved in bacteria and appears to be essential; at least we were unable to delete the gene in S. aureus. There are also prophage and plasmid encoded Lpp. The function of the prophage encoded PhiSLT is unknown. However, the plasmid encoded Lpp in USA300 encodes for a beta-lactamase (BlaZ), which was one of the first Lpp discovered in S. aureus (Nielsen and Lampen, 1982a). This gene is plasmid encoded in many other S. aureus strains such as JH1, JH9, N315, but it may also be chromosomally located as in MRSA252 or Bmb9393. The advantage of the membrane-anchored penicillinase is that it is retained at the cell surface and is not diluted in the environment like the secreted ones.

\section{Paralogous tandem lpp gene cluster}

USA300 belongs to the hypervirulent clonal complex CC8 (Robinson et al., 2005; Cockfield et al., 2007), Most of these virulent strains carry a conserved genomic island termed $\nu \mathrm{Sa} \alpha$ that encodes a number of homologous $l p p$ arranged in tandem, referred to as "lipoprotein-like" (lpl) (Babu et al., 2006). Most likely, the $l p l$ cluster represents an example of paralogous genes in $S$. aureus, which are homologs genes in this species and that have diverged after a duplication event. USA300 carries nine such $l p l$ genes. The exact activity of the Lpl proteins is unknown. However, recently it has been shown that this $l p l$ cluster triggers host cell invasion, increases pathogenicity, and it

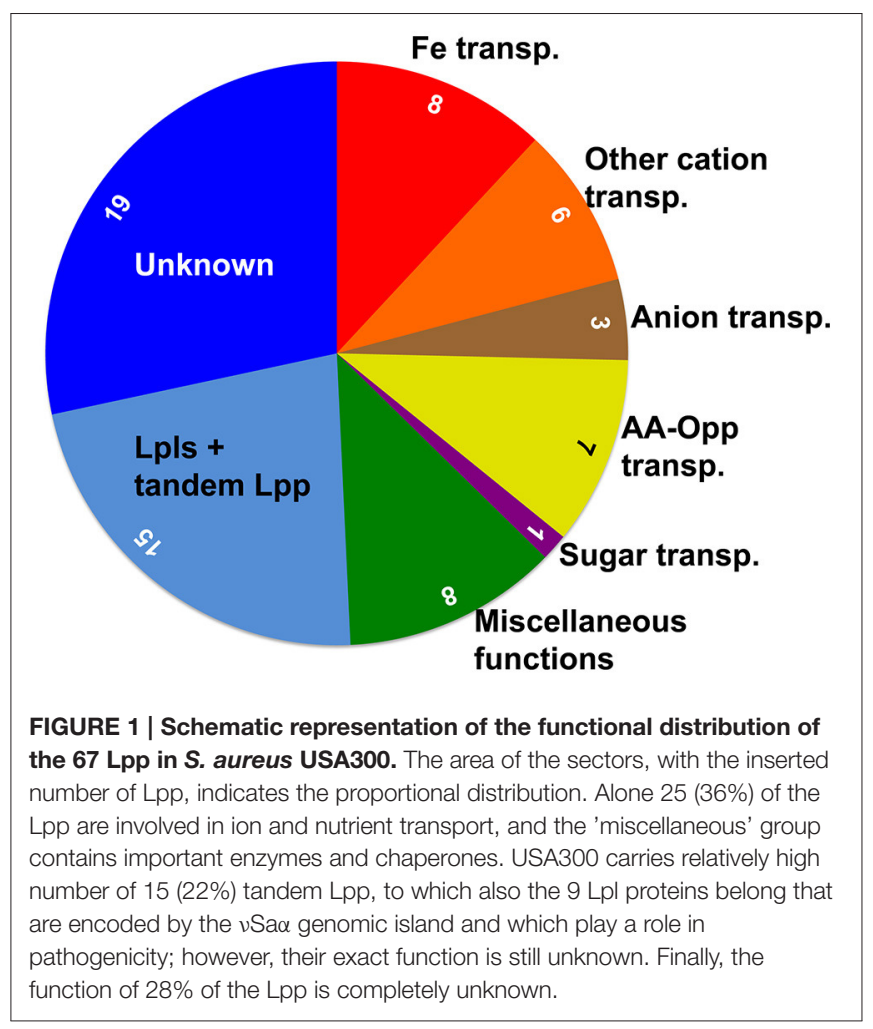

has been speculated that the $l p l$ cluster contributes to epidemic of the CC8 and CC5 strains (Nguyen et al., 2015a). Moreover, there are two other clusters of paralogous lpp genes that also contain the same conserved motifs as DUF567 of unknown function, and which are taxonomically restricted to staphylococci. With one of the paralogous Lpp CsalA the structure has been determined that revealed a new structure family (Schluepen et al., 2013).

\section{Lpp with unknown function}

Among the 67 Lpp there are 19 (28\%) with unknown function. A graphical representation of the grouped Lpp is shown in Figure 1.

\section{Dissemination of Lpp in the Genus Staphylococcus and Beyond}

We questioned how widely distributed the various Lpp are. Do they only occur in single strains, the entire species of $S$. aureus, the whole genus, or even beyond? Therefore, the amino acid sequence of each Lpp was analyzed by BLAST to identify corresponding homologs. As a cut off we have chosen $\geq 40 \%$ identity over the entire protein sequence. The comparison was restricted to those species and their representatives where a genome was available. With regards to their abundance we categorized the Lpp of USA300 into four groups: Group A comprises Lpp that were widely distributed in the entire Staphylococcus genus and in many other genera. Group B comprises Staphylococcus genus specific Lpp. Group C represent Lpp that are mainly found only in S. aureus, and Group D represents only a few Lpp that are essentially USA300 specific. 
How these 4 groups differ from each other is illustrated in Figure 2, which shows one example of each Lpp group.

The first half of the strains represent $S$. aureus strains; the underlined colored bar indicates their association to the corresponding clonal complexes (CC-types). The second halve of the strains represent a selection of other staphylococcal species; the underlined colored bar indicates whether they are regarded as semi-pathogenic or non-pathogenic. In the A and B group the USA300 Lpp are highly conserved in the S. aureus species (>95 identity) thus comprising a homogeneous block. Members of the A group are distinguished by a broad dissemination and are highly conserved in other staphylococcal species $(>70 \%$ identity) and in many other genera ( $\geq 40 \%$ identity). The group B Lpp are less conserved in other staphylococcal species and are only occasionally found in other genera. Group C lists Lpp that essentially occur in the species $S$. aureus apart from a few examples that show a slightly broader dissemination. And, finally, group D lists Lpp that are essentially strain specific for USA300 and that are not found in other $S$. aureus strains apart from few exceptions. A complete listing of all 67 Lpp is shown in Supplementary Figure 1.

Group A Lpp comprise highly conserved Lpp with a broad dissemination (Table 2A). Most likely they play an important role in metabolism or are involved in basic cellular processes. The majority of these Lpp are annotated as transporters. The corresponding homologous proteins are found in many, but not all, other staphylococcal species, and they are also found in many other genera. Examples of the most conserved Lpp that were even found in completely unrelated genera such Gram-negatives or high-GC Gram-positives are: Fe transporter-SirA (04), IsdE (05), FepA (06), MntC (SitC) (09), Phosphate ABC transporter (15), Glycine betaine transporter (18), Amino acid ABC transporter (19), D-Methionine transporter (24), Quinol oxidase (QoxA) (27), Electron transfer domain/SaeP (28), YidC (31), BlaZ (33), or the Lpp with unknown function (49). The latter Lpp is unusual in so far as it is only found in USA300 but not in other S. aureus strains; however, it is present in many other staphylococcal species and even in other genera. The most disseminated Lpp is the D-Methionine ABC transporter (24). In E. coli the MetNIQ transporter, belonging to $\mathrm{ABC}$ type permease superfamily, is involved in the uptake of both D- and L-methionine. MetN is the putative ATPase, MetI is a membrane spanning protein and MetQ (the Lpp) is the major binding protein for both L- and Dmethionine as well as their analogs, such as $\mathrm{N}$-formyl methionine (Merlin et al., 2002). In complex medium the transporter is not essential for growth, which also applies for most other Lpp. However, under certain nutrient limitations and environmental conditions they are crucial for growth and survival. So far we know only one Lpp that is essential even in complex medium, and that is YidC (31). YidC is evolutionarily conserved and is involved in membrane biogenesis in bacteria, mitochondria, and chloroplasts. It comprises several activities, such as acting as a protein insertase, as chaperone, and as an assembly factor for transmembrane proteins (Wang and Dalbey, 2011).

Group B represents Lpp that are mainly found in the genus Staphylococcus. Only exceptionally they were also found in the related genera Salinicoccus and Bacillus, or in the unrelated genus Xylanimonas, a high-GC Gram-positive cocci belonging to the Actinomycetales (Table 2B). Only 8 of the 16 Lpp in this list have an annotated function such as the ferric hydroxamate receptor (FhuD1) (01), nickel- (12), oligopeptide (Opp4A) (22) transporters, as well as thioredoxin disulfide-isomerase (30). The functions of the other $8 \mathrm{Lpp}$ are unknown.

Group C represent Lpp that are mainly found in the S. aureus species (Table 2C). Only two Lpp are ascribed a function, the iron binding protein (08) and the nickel-peptide transporter (13). Besides S. aureus, this latter Lpp is also found in various Bacillus genera, suggesting that the corresponding gene was exchanged by horizontal transfer and was maintained in $S$. aureus probably because of its beneficial effect in infection. Interestingly, all 9 Lpl proteins encoded on the $\nu \mathrm{Sa} \alpha$ island, as well as two other tandem Lpp clusters, were only found in S. aureus. These genes likely arose by gene duplication, an important mechanism for acquiring new genes and creating genetic novelty in organisms (Magadum et al., 2013). It is striking why only S. aureus accumulated and preserved these Lpl and tandem Lpp. Their contribution in virulence has been shown for the Lpl proteins; they modulate the innate immunity, and enhance host cell invasion and pathogenicity (Nguyen et al., 2015a). Finding out their precise function warrants further investigation.

Group D represents Lpp that are essentially USA300 specific and are not found in other $S$. aureus strains, but some are found in few other staphylococcal species or even in other genera as the Lpp (49) (Table 2D). The prophage encoded PhiSLT (32) is only found in USA300 and a limited number of other $S$. aureus strains as well as in S. xylosus, suggesting a limited distribution of this prophage in the genus.

\section{Correlation between the Number of Lpp and Pathogenicity}

We compared the total number of Lpp (based on PREP-LIPO method) in various $S$. aureus strains belonging to different clonal complex groups (CC 8, 5, 1, 30, 151) and in representatives of other staphylococcal species (Table 3). As can be seen, the highly epidemic categorized strains (S. aureus USA300, Newman, Mu50, Mu3, JH1, JH9, N315) contain more than 60 putative Lpp, whereas the moderate epidemic strains (S. aureus NCTC8325, S. epiderminis RP62A, ATCC_12228, S. haemolyticus JCSC1435, S. saprophyticus ATCC 15305) contain only around 50 putative Lpp. Although speculative, this suggests that epidemic/pathogenic strains are distinguished by a higher number of Lpp.

\section{Lpp As TLR2 Agonists}

It is long known that bacterial Lpp are recognized by Tolllike receptor 2 (TLR2) of the innate immune system and are sensed at very low concentrations (Zähringer et al., 2008). Dependent on the degree of acylation Lpp are recognized by different TLR2 heterodimers: Diacylated Lpp are recognized by TLR2 and TLR6 (Bulut et al., 2001; Takeuchi et al., 2001), while triacylated Lpp by TLR2 and TLR1 heterodimers (Takeda et al., 2002; Takeuchi et al., 2002). In Gram-negative bacteria Lpp biogenesis consists of three successive reactions catalyzed by prolipoprotein diacylglyceryl transferase (Lgt), signal peptidase II (Lsp), and apolipoprotein $\mathrm{N}$-acyltransferase (Lnt) (Sankaran and 


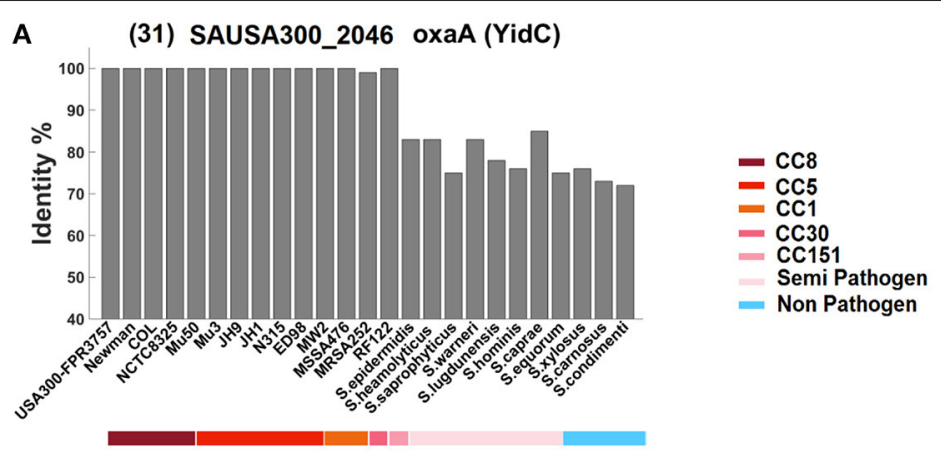

(30) SAUSA300_2354 PDI
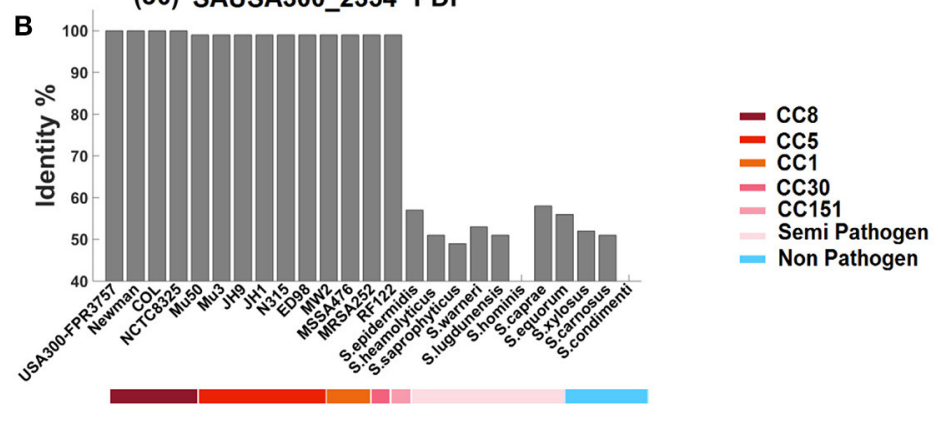

C (13) SAUSA300_0203 Ni/Pep transp
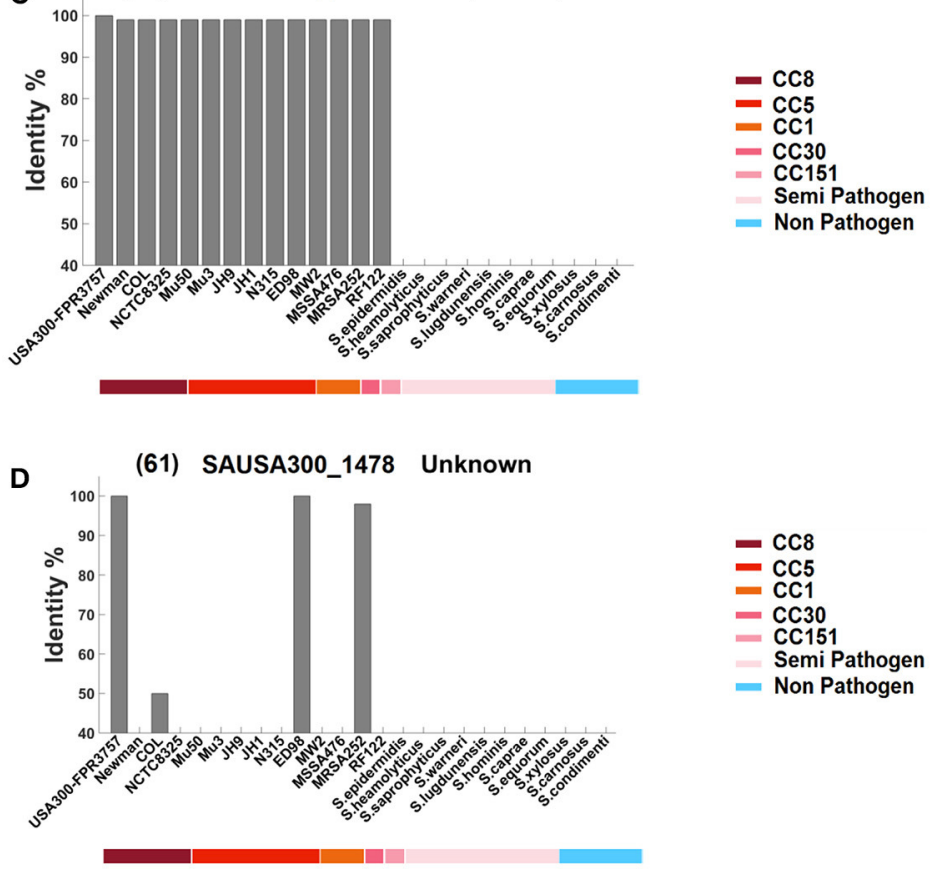

FIGURE 2 | Classification of the Lpp into four groups based on their similarity and dissemination in bacteria. Each Lpp was blasted against the indicated S. aureus strains and other staphylococcal species representatives. The cut off was $\geq 40 \%$ identity over the entire protein sequence. The colored bar below the listed strains indicates clonal complexes (CC-types) of S. aureus (first half), as well as other staphylococcal species representatives, grouped in semi-pathogenic and non-pathogenic (second half). Group (A) represents Lpp that are highly conserved in the Staphylococcus genus but also in many other genera; an example for this group is YidC, an essential protein in many bacteria. Group (B) represents Lpp that are mainly found in the genus Staphylococcus; the example for this group is PDI, a proposed thioredoxin disulfide-isomerase. Group (C) represents Lpp mainly found in the S. aureus species; an example is the proposed nickel-peptide transporter. Group (D) represents strain-specific Lpp essentially occurring in the strain USA300; the example is an unknown Lpp. The number in front of the gene ID refers to the corresponding numbering in Table 1. 
TABLE 2A | USA300 Lpp with broad dissemination.

\begin{tabular}{|c|c|c|}
\hline No $^{1}$ & Function/annotation & Staphylococcal species and other genera 2 \\
\hline 02 & Fe ABC transporter/FhuD2 & $\begin{array}{l}\text { S.aureus, S.capitis, S.carnosus, S.equorum, S.gallinarum, S.haemolyticus, S.hyicus, S.lugdunensis, S.pasteuri, } \\
\text { S.pettenkoferi, S.pseudintermedius, S.saprophyticus, S.schleiferi, S.simiae, S.warneri, } \\
\text { S.xylosus, } \\
\text { Bacillus, Exiguobacterium, Macrococcus, Salinicoccus }\end{array}$ \\
\hline 04 & Fe ABC transporter/SirA & $\begin{array}{l}\text { S.aureus, S.agnetis, S.arlettae, S.delphini, S.equorum, S.hyicus, S.intermedius, S.lugdunensis, } \\
\text { S.pseudintermedius, S.schleiferi }\end{array}$ \\
\hline
\end{tabular}

$05 \quad$ Fe ABC transporter/IsdE

06 FepA, Fe-binding protein, part of fep ABC and tat-AC cluster

Manganese-binding protein MntC (SitC)

Zinc-binding, adcA-like

Phosphate ABC transporter

Phosphonate ABC transporter

Nitrate ABC transporter

Glycine betaine /carnitine/choline ABC transporter (OpuCc)
Advenella, Bacillus, Chromohalobacter, Clostridium, Gynuella, Hahella, Halobacteroides, Halomonas, Haloplasma, Jeotgalibacillus, Lysinibacillus, Marinomonas, Paenibacillus, Pectobacterium, Planococcus, Sporosarcina

S.aureus, S.auricularis, S.capitis, S.caprae, S.condimenti, S.lentus, S.Iugdunensis, S.pasteuri, S.sciuri, S.simulans Bacillus, Carnobacterium, Coprococcus, Eubacterium, Lactobacillus, Listeria, Lysinibacillus, Paenibacillus, Solibacillus, Streptococcus, Terribacillus

S.aureus, S.capitis, S.carnosus, S.condimenti, S.epidermidis, S.haemolyticus, S.lugdunensis, S.pasteuri, S. simiae, S.warneri

Actinobacillus, Actinoplanes, Bacillus, Bibersteinia, Brevibacillus, Clavibacter, Corynebacterium, Dermacoccus, Exiguobacterium, Gallibacterium, Hyphomicrobium, Kineococcus, Kitasatospora, Kyrpidia, Leptotrichia, Listeria, Lysinibacillus, Mannheimia, Moraxella, Mycobacterium, Neisseria, Paenibacillus, Planococcus, Pseudomonas, Streptococcus, Streptomyces, Yersinia

S.aureus, S.capitis, S.caprae, S.carnosus, S.condimenti, S.epidermidis, S.equorum, S.haemolyticus, S.hominis, S.hyicus, S.lugdunensis, S.pasteuri, S.pseudintermedius, S.saprophyticus, S.schleiferi, S.simiae, S.simulans, S.warneri, S.xylosus

Aerococcus, Amphibacillus, Bacillus, Carnobacterium, Clostridium, Enterococcus, Exiguobacterium, Finegoldia, Lactobacillus, Macrococcus, Oceanobacillus, Paenibacillus, Streptomyces, Terribacillus, Tetragenococcus

S.aureus, S.capitis, S.haemolyticus, S.hyicus, S.pasteuri, S.pseudintermedius, S.saprophyticus, S.schleiferi,

S.warneri, S.xylosus

Bacillus, Enterococcus, Halobacillus, Streptococcus

S.aureus, S.epidermidis, S. hyicus, S.lugdunensis, S.pasteuri, S.pseudintermedius, S. saprophyticus, S.schleiferi, S.warneri, S.xylosus,

Aggregatibacter, Bacillus, Brevibacillus, Eubacterium, Mannheimia, Methanosarcina, Paenibacillus, Proteus

S.aureus, S.capitis, S.caprae, S.carnosus, S.epidermidis, S.haemolyticus, S.hyicus, S.lugdunensis, S.pasteuri, S.pseudintermedius, S. saprophyticus, S.schleiferi, S.simiae, S.warneri, S.xylosus

Bacillus, Clostridium, Macrococcus, Paenibacillus, Salinicoccus, Syntrophobotulus

S.aureus, S.capitis, S.caprae, S.carnosus, S.epidermidis, S.haemolyticus, S.hyicus, S.lugdunensis, S.pasteuri, S.pseudintermedius, S.saprophyticus, S.schleiferi, S.simiae, S.warneri, S.xylosus

Alkaliphilus, Anoxybacillus, Bacillus, Clostridium, Enterobacter, Escherichia, Eubacterium, Exiguobacterium, Geobacillus, Halobacillus, Jeotgalibacillus, Lysinibacillus, Macrococcus, Maribacter, Paenibacillus, Planococcus, Pleurocapsa, Salinicoccus, Sebaldella, Streptomyces, Thermobacillus, Thermosediminibacter

S.aureus, S.capitis, S.caprae, S.epidermidis, S.haemolyticus, S.hominis, S.lugdunensis, S.pasteuri, S. saprophyticus, S.warneri

Bacillus, Carnobacterium, Enterobacter, Enterococcus, Lactobacillus, Melissococcus, Paenibacillus, Terribacillus, Weissella

S.aureus, S.pseudintermedius, S.saprophyticus, S.schleiferi

Bacillus, Clostridium, Geobacillus, Methanosarcina, Ruminiclostridium, Solibacillus

S.aureus, S.capitis, S.carnosus, S.cohnii, S.condimenti, S.epidermidis, S.equorum, S.gallinarum, S.haemolyticus, S.hyicus, S.lugdunensis, S.pasteuri, S.saprophyticus, S.schleiferi, S.simiae, S.simulans, S.succinus, S.warneri, S.xylosus

Bacillus, Carnobacterium, Enterococcus, Lactobacillus, Listeria, Macrococcus, Pediococcus, Solibacillus, Streptococcus, Tetragenococcus, Virgibacillus

S.aureus, S.capitis, S.caprae, S.carnosus, S.epidermidis, haemolyticus, S.lugdunensis, S.pasteuri, S.saprophyticus, S.simiae, S.warneri, S. S.xylosus

Aggregatibacter, Anoxybacillus, Arthrobacter, Bacillus, Brevibacillus, Brucella, Campylobacter, Clostridium, Ensifer, Gallibacterium, Geobacillus, Haemophilus, Hafnia, Halobacillus, Jeotgalibacillus, Macrococcus, Mesorhizobium, Neisseria, Neorhizobium, Nocardia, Ochrobactrum, Paenibacillus, Photorhabdus, Raoultella, Rhizobium, Sinorhizobium, Streptococcus, Xenorhabdus 


\section{TABLE 2A | Continued}

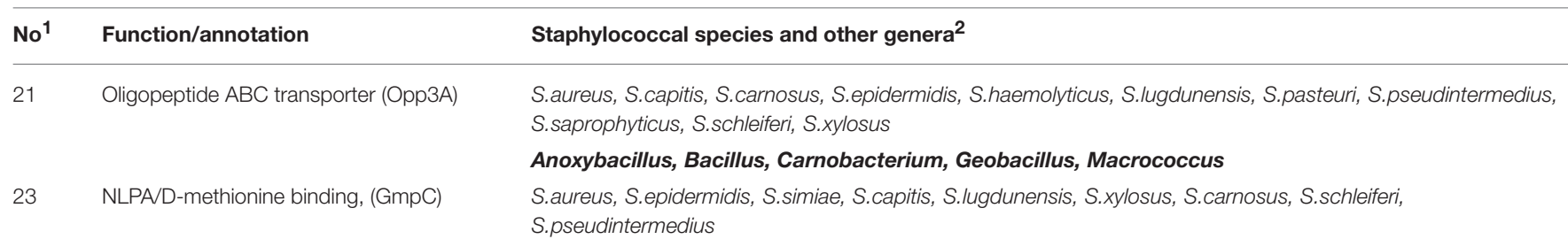

Aerococcus, Amphibacillus, Bacillus, Carnobacterium, Clostridium, Enterococcus, Exiguobacterium, Finegoldia, Lactobacillus, Listeria, Macrococcus, Megasphaera, Oceanobacillus, Paenibacillus, Ralstonia, Streptococcus, Streptomyces, Terribacillus, Tetragenococcus

(a)

S.aureus, S.capitis, S.caprae, S.carnosus, S.cohnii, S.epidermidis, S.haemolyticus, S.hyicus, S.lugdunensis, S.pasteuri, S.pseudintermedius, S.saprophyticus, S.schleiferi, S.warneri, S.xylosus

Acetohalobium, Acidaminococcus, Actinoplanes, Advenella, Amphibacillus, Anoxybacillus, Arcobacter, Azospirillum, Bacillus, Bifidobacterium, Caldicellulosiruptor, Campylobacter, Carnobacterium, Cedecea, Citrobacter, Clostridium, Cronobacter, Desulfitobacterium, Desulfosporosinus, Enterobacter, Enterobacteriaceae, Enterococcus, Enwinia, Exiguobacterium, Finegoldia, Geobacillus, Halobacillus, Jeotgalibacillus, Ketogulonicigenium, Klebsiella, Kluyvera, Kosakonia, Lactobacillus, Leptotrichia, Lipotes, Listeria, Macrococcus, Megamonas, Megasphaera, Melissococcus, Paenibacillus, Pectobacterium, Pediococcus, Pelosinus, Planococcus, Pseudomonas, Salinicoccus, Sebaldella, Selenomonas, Serratia, Sodalis, Solibacillus, Streptomyces, Sulfurospirillum, Terribacillus, Thermoanaerobacterium, Veillonella, Virgibacillus, Xanthomonas

25 Maltose ABC transporter
$26 \quad$ CamS sex pheromone biosynthesis
$27 \quad$ Quinol oxidase, subunit II (QoxA)

27 Quinol oxidase, subunit II (QoxA)

28 Electron transfer domain/SaeP

$31 \quad$ YidC (OxaA) - essential protein
S.aureus, S.delphini, S.intermedius, S.lentus, S.pseudintermedius, S.sciuri

\section{Bacillus, Geobacillus, Salinicoccus}

S.aureus, S.capitis, S.carnosus, S.epidermidis, S. haemolyticus, S.hyicus, S.Iugdunensis, S.pasteuri, S.pseudintermedius, S.saprophyticus, S.schleiferi, S.simiae, S.warneri, S.xylosus

\section{Escherichia, Macrococcus, Salinicoccus}

S.aureus, S.agnetis, S.auricularis, S.capitis, S.caprae, S.carnosus, S.chromogenes, S.cohnii, S.epidermidis, S.haemolyticus, S.hominis, S.hyicus, S.lugdunensis, S.pasteuri, S.pettenkoferi, S.pseudintermedius, S.saprophyticus, S.schleiferi, S.simiae, S.warneri, S.xylosus,

Bacillus, Geobacillus, Halobacillus, Listeria, Lysinibacillus, Paenibacillus, Salinicoccus, Streptococcus, Virgibacillus

S.aureus, S.auricularis, S.capitis, S.caprae, S.carnosus, S.condimenti, S.delphini, S.epidermidis, S. haemolyticus, S.intermedius, S.lentus, S.lugdunensis, S.microti, S.pettenkoferi, S.pseudintermedius, S. saprophyticus, S.schleiferi, S.sciuri, S.simiae, S.simulans, S.vitulinus, S.xylosus

Bacillus, Brochothrix, Carnobacterium, Enterococcus, Fictibacillus, Listeria, Mycobacterium, Paenibacillus

S.aureus, S.arlettae, S.capitis, S.caprae, S.carnosus, S.cohnii, S.condimenti, S.delphini, S.epidermidis, S.equorum, S.gallinarum, S. haemolyticus, S. hominis, S.hyicus, S.lugdunensis, S.massiliensis, S. pasteuri, S.pseudintermedius, S.saprophyticus, S.schleiferi, S.simiae, S.simulans, S.succinus, S.warneri, S.xylosus

Bacillus, Halobacillus, Lysinibacillus, Macrococcus, Planococcus, Salinicoccus, Solibacillus, Xylanimonas

S.aureus, S.agnetis, S.capitis, S.epidermidis, S.equorum, S.haemolyticus, S.lugdunensis, S.pseudintermedius, S. saprophyticus, S.warneri, S.xylosus,

Acidaminococcus, Bacillus, Carnobacterium, Enterococcus, Escherichia, Kribbella, Macrococcus, Paenibacillus, Salinicoccus, Streptococcus, Streptomyces

USA300, S.arlettae, S.capitis, S.carnosus, S.cohnii, S.epidermidis, S.equorum, S.haemolyticus, S.hominis, S.Iugdunensis, S.pasteuri, S.pettenkoferi, S.pseudintermedius, S. saprophyticus, S. sciuri, S. warneri, S.xylosus

Bacillus, Carnobacterium, Corynebacterium, Dermacoccus, Enterococcus, Exiguobacterium, Geobacillus, Jeotgalibacillus, Kocuria, Kytococcus, Listeria, Lysinibacillus, Macrococcus, Oceanobacillus, Paenibacillus, Planococcus, Salinicoccus, Solibacillus, Streptococcus, Terribacillus, Virgibacillus (see Table 2D)

S.aureus, S.agnetis, S.capitis, S.haemolyticus, S.hyicus, S.simiae,

Bacillus, Clostridium, Enterococcus, Herbinix, Lactobacillus, Leuconostoc, Streptococcus

Numbering and proposed function are the same as listed in Table 1.

The other genera are in bold letters.

Wu, 1994; Buddelmeijer, 2015). A Lnt homolog has so far not been found in staphylococci. However, Kurokawa and colleagues showed that $\mathrm{MntC}(\mathrm{SitC})$ is triacylated in exponential growth phase but becomes diacylated (lacking the alpha-aminoacylation) in post-exponential phase (Kurokawa et al., 2009, 2012). The occurrence of triacylated $\mathrm{MntC}$ (SitC) indicates that S. aureus 


\section{TABLE 2B | USA300 Lpp mainly occurring in the genus Staphylococcus.}

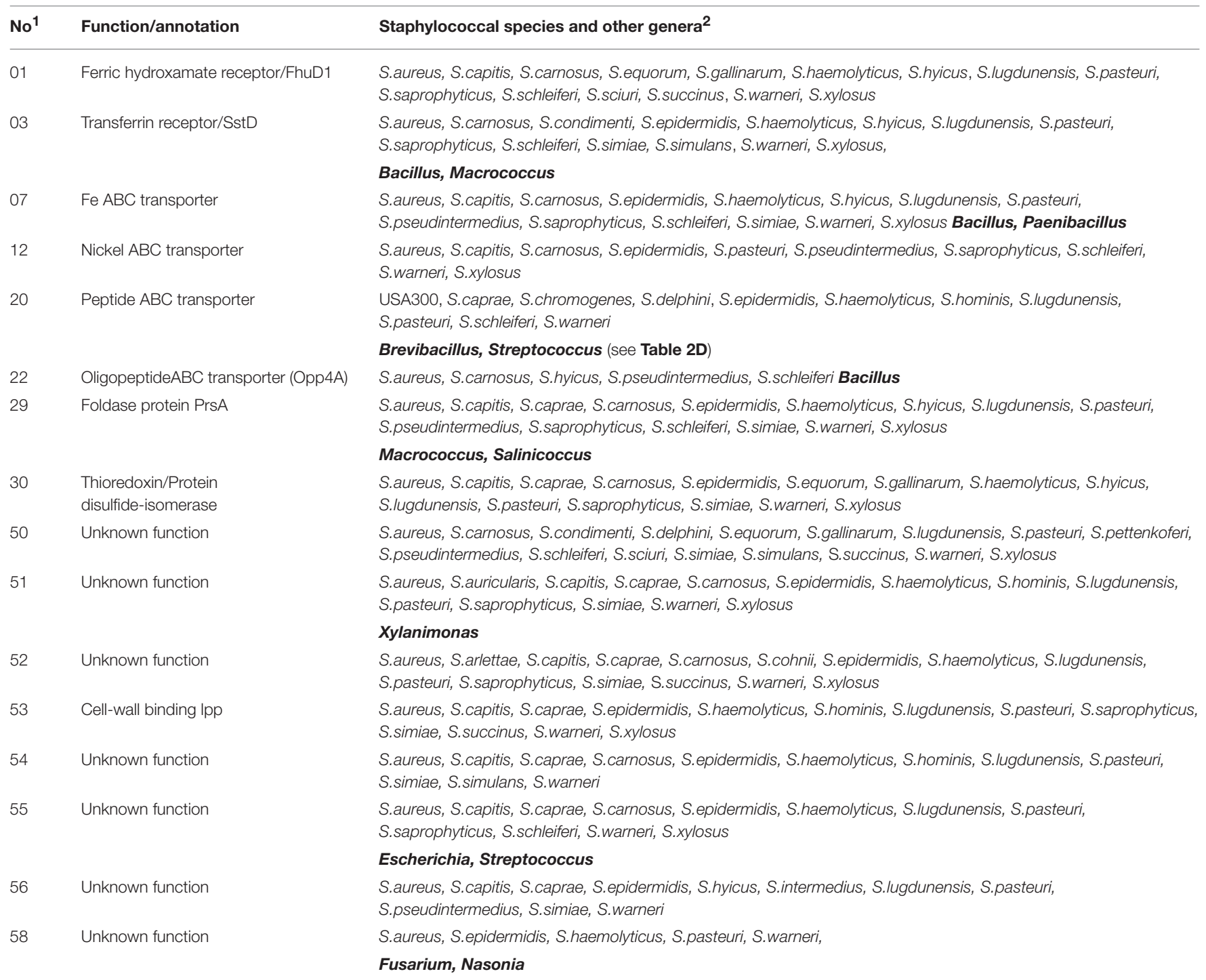

Numbering and proposed function are the same as listed in Table 1.

The other genera are in bold letters.

has a apolipoprotein $\mathrm{N}$-acyltransferase, which adds a fatty acid to the amino group of $S$-(diacylglyceryl) cysteine residue. MntC (SitC) was also the first native Lpp for which its TLR2 activation and its co-localization with TLR2 have been demonstrated (Kurokawa et al., 2009; Müller et al., 2010). The structure of the lipid moiety of Lpp has an enormous influence on the immune response. For example it has been shown that di- but not tri-acylated Lpp suppress immune responses and might play a role in immune tolerance (Skabytska et al., 2014).

\section{Lpp As Vaccine Candidates}

In principle all Lpp listed in Table $\mathbf{1}$ are potential vaccine candidates, however, some are more or less suitable. All Lpp listed in Table 2C are promising vaccine candidates because they occur essentially in the $S$. aureus species. Promising vaccine candidates would be the $9 \mathrm{Lpl}$ proteins (34-42 in Table 1) because they share a highly conserved ( $92 \%$ similarity) core region of 44 amino acids (Nguyen et al., 2015a). A vaccine against this core domain would simultaneously cross-react with several antigens. There is also a set of 4 tandem Lpp (45-48 in Table 1) that show a very high similarity $(>80 \%)$ over the entire protein length; most likely these tandem Lpp arose by gene duplication. However, both these tandem Lpp and Lpl share also a conserved 38 amino acid long domain Figure 3. Therefore, one can expect that antibodies against this conserved domain would cross-react with $13 \mathrm{Lpp}$. The advantage of these tandem Lpp is also that they are highly present only in $S$. aureus. A vaccine would therefore not target staphylococcal skin residents such as S. epidermidis and other species. Another parameter for a promising Lpp-antigen is its size. The size should be large enough that the protein part can penetrate the cell wall to be accessible for antibodies. The size of 
TABLE 2C | USA300 Lpp essentially occurring in the S. aureus species.

\begin{tabular}{|c|c|c|}
\hline $\mathrm{No}^{1}$ & Function/annotation & S. aureus and few other species/genera ${ }^{2}$ \\
\hline 08 & Iron Binding Protein & $\begin{array}{l}\text { S.aureus, S. epidermidis, S.pseudintermedius, } \\
\text { S.schleiferi }\end{array}$ \\
\hline \multirow[t]{2}{*}{13} & Nickel-Peptide/transporter & S. aureus only - but also in: \\
\hline & & $\begin{array}{l}\text { Amphibacillus, Bacillus, Lysinibacillus, } \\
\text { Solibacillus }\end{array}$ \\
\hline 34 & Lpl-1 vSa $\alpha$ specific & S.aureus \\
\hline 35 & Lpl-2 vSa $\alpha$ specific & S.aureus \\
\hline 36 & Lpl-3 vSa $\alpha$ specific & S.aureus \\
\hline 37 & Lpl-4 vSa $\alpha$ specific & S.aureus \\
\hline 38 & Lpl-5 vSa $\alpha$ specific & S.aureus \\
\hline 39 & Lpl-6 vSa $\alpha$ specific & S.aureus \\
\hline 40 & Lpl-7 vSa $\alpha$ specific & S.aureus \\
\hline 41 & Lpl-8 vSa $\alpha$ specific & S.aureus \\
\hline 42 & Lpl-9 vSa $\alpha$ specific & S.aureus \\
\hline 43 & Tandem Ipp & S.aureus, S.heamolyticus, S.lugdunensis \\
\hline 44 & Tandem Ipp & S.aureus \\
\hline 45 & Tandem Ipp (Csa1A) & S.aureus \\
\hline 46 & Tandem Ipp & S.aureus \\
\hline 47 & Tandem Ipp & S.aureus \\
\hline 48 & Tandem Ipp & S.aureus \\
\hline 59 & Unknown function & $\begin{array}{l}\text { S.aureus, S.epidermidis, S.Iugdunensis } \\
\text { S.pasteuri, S.warneri }\end{array}$ \\
\hline 60 & Unknown function & $\begin{array}{l}\text { S.aureus, S.caprae, S.epidermidis, } \\
\text { S.lugdunensis, S.warneri }\end{array}$ \\
\hline 62 & Unknown function & S.aureus, S.epidermidis \\
\hline 63 & Unknown function & S.aureus, S.epidermidis \\
\hline 64 & Unknown function & S.aureus, S.epidermidis \\
\hline 65 & Unknown function & S.aureus, S.epidermidis \\
\hline \multirow[t]{2}{*}{66} & Unknown function & S.aureus \\
\hline & & Bacillus \\
\hline 67 & Unknown function & S.aureus \\
\hline
\end{tabular}

Numbering and proposed function are the same as listed in Table 1.

The other genera are in bold letters.

\section{TABLE 2D | USA300 Lpp essentially occurring in strain USA300.}

\begin{tabular}{lll}
\hline 20 & Peptide ABC transporter & $\begin{array}{l}\text { Only USA300 not in other } \text { S. aureus strains, but } \\
\text { Other species and genera (see Table 2B) } \\
\text { Only USA300, ED98, MW2, MSSA476, } \\
\text { MRSA252 and S.xylosus }\end{array}$ \\
$49 \begin{array}{l}\text { PhiSLT ORF144-like } \\
\text { Onknown function }\end{array}$ & $\begin{array}{l}\text { Other Species and genera (see Table 2A) } \\
\text { Only USA300, COL, ED98, MRSA252 }\end{array}$ \\
61 Unknown function & Only
\end{tabular}

the Lpp varies enormously, ranging from 6 to $89 \mathrm{kDa}$ (Table 1). All Lpp with a mass $>40 \mathrm{kDa}$ are also good candidates like the zinc-binding (10), cobalt and nickel transporter Cnt (11), nickel ABC transporter (12), nickel-Peptide/ transporter (13), peptide $\mathrm{ABC}$ transporter (20), oligopeptide $\mathrm{ABC}$ transporter (Opp3A) (21), CamS sex pheromone biosynthesis (26), quinol oxidase, subunit II (QoxA) (27).
TABLE 3 | The number of Lpp in different staphylococcal strains and species.

\begin{tabular}{llll}
\hline Species & & Strains & Number of Lpp \\
\hline S.aureus & CC8 & USA300 & 67 \\
& & Newman & 64 \\
& & COL & 61 \\
& CC5 & NCTC8325 & 50 \\
& & Mu50 & 66 \\
& Mu3 & 66 \\
& & JH1 & 65 \\
& & JH9 & 65 \\
& N315 & 64 \\
S. epidermidis & CC1 & MSSA476 & 62 \\
S. haemolyticus & MW2 & 60 \\
S. saprophyticus & CC30 & MRSA252 & 57 \\
S. carnosus & & RF122 & 56 \\
\hline
\end{tabular}

A third parameter for a promising vaccine candidate is the abundance of an antigen. The most abundant Lpp is MntC ( $\mathrm{SitC}$ ), which is essential for MRSA virulence during murine systemic infection (Kehl-Fie et al., 2013). It was therefore concluded that MntABC might be a potential vaccine candidate (Diep et al., 2014). Another potential vaccine candidate is the FhuD2 (02) involved in ferric-hydroxamate uptake. FhuD2 binds ferrichrome with nanomolar affinity and the structure of FhuD2-ferrichrome has been determined (Mariotti et al., 2013; Podkowa et al., 2014). Immunization with FhuD2 alone or together with hydroxamate siderophores was protective in a murine staphylococcal infection model (Mariotti et al., 2013). However, a break-through was reported recently with a combination of five antigens, including FhuD2 (02), and Csa1A (45), formulated with a novel adjuvant containing a TLR7-dependent agonist adsorbed to alum. This vaccine provided close to $100 \%$ protection against four different staphylococcal strains (Bagnoli et al., 2015). As can seen, certain Lpp have been already turned out experimentally as promising vaccine candidates.

\section{CONCLUSION}

The re-evaluation of the chromosomal encoded Lpp in S. aureus USA300 was necessary, as none of the Lpp prediction tools of the public domain yielded the complete inventory of Lpp. The 67 identified Lpp constituted a solid basis for a systematic analysis. A large proportion of the Lpp is involved in the uptake of essential ions and nutrients. Frequently they function as receptors for the target molecule and are part of $\mathrm{ABC}$ transporter complexes. Generally one can say that 39\% of the Lpp are involved in ion and nutrient transport, indicating, that this is one of their major physiological task. However, two Lpp in the miscellaneous group 


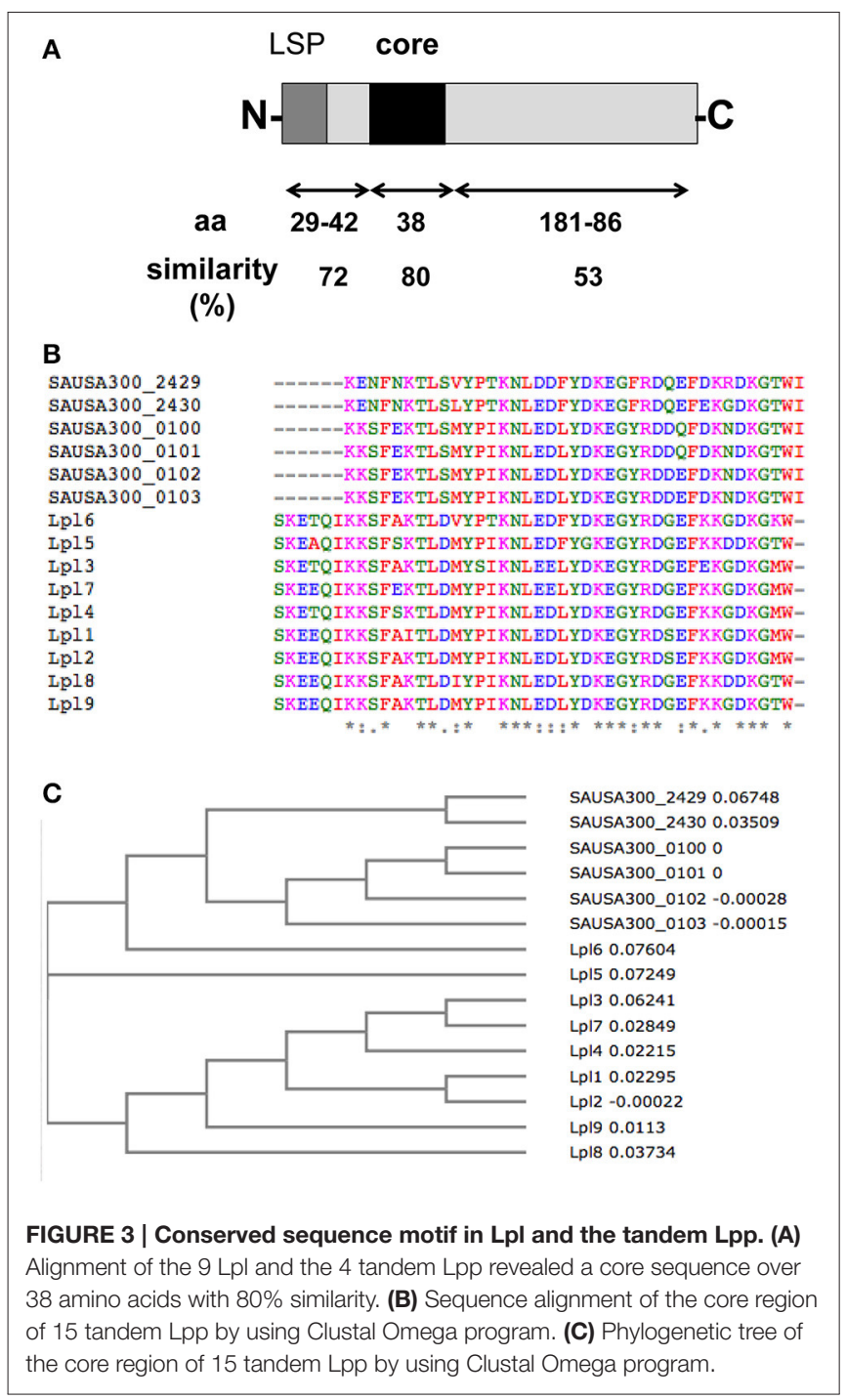

should be mentioned, QoxA and YidC, which play important functions in respiration and folding of membrane proteins.

Regarding the dissemination of Lpp within the S. aureus species and in other species we can group them into

\section{REFERENCES}

Babu, M. M., Priya, M. L., Selvan, A. T., Madera, M., Gough, J., Aravind, L., et al. (2006). A database of bacterial lipoproteins (DOLOP) with functional assignments to predicted lipoproteins. J. Bacteriol. 188, 2761-2773. doi: 10.1128/Jb.188.8.2761-2773.2006

Bagnoli, F., Fontana, M. R., Soldaini, E., Mishra, R. P., Fiaschi, L., Cartocci, E., et al. (2015). Vaccine composition formulated with a novel TLR7-dependent adjuvant induces high and broad protection against Staphylococcus aureus. Proc. Natl. Acad. Sci. U.S.A. 112, 3680-3685. doi: 10.1073/pnas.1424924112

Bagos, P. G., Tsirigos, K. D., Liakopoulos, T. D., and Hamodrakas, S. J. (2008). Prediction of lipoprotein signal peptides in Gram-positive bacteria with a Hidden Markov Model. J. Proteome Res. 7, 5082-5093. doi: 10.1021/pr800162c four categories: (i) those that are highly conserved and broadly disseminated, (ii) that are mainly found in the genus Staphylococcus, (iii) that are mainly found in the species $S$. aureus, and (iv) a few Lpp are rather strain specific. Particularly the group of $S$. aureus specific Lpp are promising vaccine candidates for future work. Our data show that the relatively high number of $l p p$ genes $(>60)$ in the $S$. aureus species is mainly due to the tandem lpp. There was only one $S$. aureus strain, NCTC8325, that shows only $50 \mathrm{lpp}$. As this strain is used for almost 50 years as a lab strain, it is conceivable that under non-selective conditions a reversal of the lpp amplification occurred. Indeed, this strain contains only three tandem $l p l$ genes in the $\nu \mathrm{Sa} \alpha$ island, which might represent an example of adaptive evolution. There are many open questions regarding the Lpp in S. aureus and also the Lpp of the staphylococcal skin microbiota that need to be studied in the future.

\section{AUTHOR CONTRIBUTIONS}

FG designed the work, SS and MN analyzed and interpreted data, SS and MN prepared the draft of the Manuscript (MS), figures and tables; and FG wrote the MS.

\section{FUNDING}

This work was supported by grants from the Deutsche Forschungsgemeinschaft (DFG; GO 371/9-1, SFB766 and TRR34) and Open Access Publishing Fund of Tuebingen University.

\section{ACKNOWLEDGMENTS}

We would like to thank Dr. Ralf Rosenstein for his useful suggestion and discussion.

\section{SUPPLEMENTARY MATERIAL}

The Supplementary Material for this article can be found online at: http://journal.frontiersin.org/article/10.3389/fmicb. 2016.01404 
Buddelmeijer, N. (2015). The molecular mechanism of bacterial lipoprotein modification-how, when and why? FEMS Microbiol. Rev. 39, 246-261. doi: $10.1093 /$ femsre/fuu006

Bulut, Y., Faure, E., Thomas, L., Equils, O., and Arditi, M. (2001). Cooperation of Toll-like receptor 2 and 6 for cellular activation by soluble tuberculosis factor and Borrelia burgdorferi outer surface protein A lipoprotein: role of Toll-interacting protein and IL-1 receptor signaling molecules in Toll-like receptor 2 signaling. J. Immunol. 167, 987-994. doi: 10.4049/jimmunol.16 7.2.987

Cockayne, A., Hill, P. J., Powell, N. B., Bishop, K., Sims, C., and Williams, P. (1998). Molecular cloning of a 32-kilodalton lipoprotein component of a novel iron-regulated Staphylococcus epidermidis ABC transporter. Infect. Immun. 66, 3767-3774.

Cockfield, J. D., Pathak, S., Edgeworth, J. D., and Lindsay, J. A. (2007). Rapid determination of hospital-acquired meticillin-resistant Staphylococcus aureus lineages. J. Med. Microbiol. 56(Pt 5), 614-619. doi: 10.1099/jmm.0.47074-0

Dale, S. E., Sebulsky, M. T., and Heinrichs, D. E. (2004). Involvement of SirABC in iron-siderophore import in Staphylococcus aureus. J. Bacteriol. 186, 8356-8362. doi: 10.1128/jb.186.24.8356-8362.2004

Diep, B. A., Gill, S. R., Chang, R. F., Phan, T. H., Chen, J. H., Davidson, M. G., et al. (2006). Complete genome sequence of USA300, an epidemic clone of community-acquired meticillin-resistant Staphylococcus aureus. Lancet 367, 731-739. doi: 10.1016/S0140-673668231-7

Diep, B. A., Phung, Q., Date, S., Arnott, D., Bakalarski, C., Xu, M., et al. (2014). Identifying potential therapeutic targets of methicillin-resistant Staphylococcus aureus through in vivo proteomic analysis. J. Infect. Dis. 209, 1533-1541. doi: 10.1093/infdis/jit662

Gan, K., Sankaran, K., Williams, M. G., Aldea, M., Rudd, K. E., Kushner, S. R., et al. (1995). The umpA gene of Escherichia coli encodes phosphatidylglycerol:prolipoprotein diacylglyceryl transferase (lgt) and regulates thymidylate synthase levels through translational coupling. $J$. Bacteriol. 177, 1879-1882.

Götz, F., and Mayer, S. (2013). Both terminal oxidases contribute to fitness and virulence during organ-specific Staphylococcus aureus colonization. MBio 4, e00976-e00913. doi: 10.1128/mBio.00976-13

Grigg, J. C., Vermeiren, C. L., Heinrichs, D. E., and Murphy, M. E. (2007). Heme coordination by Staphylococcus aureus IsdE. J. Biol. Chem. 282, 28815-28822. doi: 10.1074/jbc.M704602200

Hamilton, A., Robinson, C., Sutcliffe, I. C., Slater, J., Maskell, D. J., DavisPoynter, N., et al. (2006). Mutation of the maturase lipoprotein attenuates the virulence of Streptococcus equi to a greater extent than does loss of general lipoprotein lipidation. Infect. Immun. 74, 6907-6919. doi: 10.1128/IAI.01 116-06

Hammer, N. D., Reniere, M. L., Cassat, J. E., Zhang, Y., Hirsch, A. O., Indriati Hood, M., et al. (2013). Two heme-dependent terminal oxidases power Staphylococcus aureus organ-specific colonization of the vertebrate host. MBio 4:e00241-13. doi: 10.1128/mBio.00241-13

Hantke, K., and Braun, V. (1973). Covalent binding of lipid to protein. Diglyceride and amide-linked fatty acid at the $\mathrm{N}$-terminal end of the murein-lipoprotein of the Escherichia coli outer membrane. Eur. J. Biochem. 34, 284-296.

Heikkinen, O., Seppala, R., Tossavainen, H., Heikkinen, S., Koskela, H., Permi, P., et al. (2009). Solution structure of the parvulin-type PPIase domain of Staphylococcus aureus PrsA-implications for the catalytic mechanism of parvulins. BMC Struct. Biol. 9:17. doi: 10.1186/1472-6807-9-17

Heinrichs, J. H., Gatlin, L. E., Kunsch, C., Choi, G. H., and Hanson, M. S. (1999). Identification and characterization of SirA, an iron-regulated protein from Staphylococcus aureus. J. Bacteriol. 181, 1436-1443.

Henneke, P., Dramsi, S., Mancuso, G., Chraibi, K., Pellegrini, E., Theilacker, C., et al. (2008). Lipoproteins are critical TLR2 activating toxins in group B streptococcal sepsis. J. Immunol. 180, 6149-6158. doi: 10.4049/jimmunol.180.9.6149

Hiron, A., Borezée-Durant, E., Piard, J. C., and Juillard, V. (2007). Only one of four oligopeptide transport systems mediates nitrogen nutrition in Staphylococcus aureus. J. Bacteriol. 189, 5119-5129. doi: 10.1128/jb.00274-07

Horsburgh, M. J., Wharton, S. J., Cox, A. G., Ingham, E., Peacock, S., and Foster, S. J. (2002). MntR modulates expression of the PerR regulon and superoxide resistance in Staphylococcus aureus through control of manganese uptake. Mol. Microbiol. 44, 1269-1286. doi: 10.1046/j.1365-2958.2002.02944.x
Hussain, M., Ichihara, S., and Mizushima, S. (1982). Mechanism of signal peptide cleavage in the biosynthesis of the major lipoprotein of the Escherichia coli outer membrane. J. Biol. Chem. 257, 5177-5182.

Jeong, D. W., Cho, H., Jones, M. B., Shatzkes, K., Sun, F., Ji, Q., et al. (2012). The auxiliary protein complex SaePQ activates the phosphatase activity of sensor kinase SaeS in the SaeRS two-component system of Staphylococcus aureus. Mol. Microbiol. 86, 331-348. doi: 10.1111/j.1365-2958.2012.08198.x

Jousselin, A., Manzano, C., Biette, A., Reed, P., Pinho, M., Rosato, A., et al. (2015). The Staphylococcus aureus chaperone PrsA is a new auxiliary factor of oxacillin resistance affecting Penicillin-binding protein 2A. Antimicrob. Agents Chemother. 60, 1656-1666. doi: 10.1128/AAC.02333-15

Jousselin, A., Renzoni, A., Andrey, D. O., Monod, A., Lew, D. P., and Kelley, W. L. (2012). The posttranslocational chaperone lipoprotein PrsA is involved in both glycopeptide and oxacillin resistance in Staphylococcus aureus. Antimicrob. Agents Chemother. 56, 3629-3640. doi: 10.1128/AAC.06264-11

Juncker, A. S., Willenbrock, H., Von Heijne, G., Brunak, S., Nielsen, H., and Krogh, A. (2003). Prediction of lipoprotein signal peptides in Gram-negative bacteria. Protein Sci. 12, 1652-1662. doi: 10.1110/ps.0303703

Kehl-Fie, T. E., Zhang, Y., Moore, J. L., Farrand, A. J., Hood, M. I., Rathi, S., et al. (2013). MntABC and MntH contribute to systemic Staphylococcus aureus infection by competing with calprotectin for nutrient manganese. Infect. Immun. 81, 3395-3405. doi: 10.1128/IAI.00420-13

Khandavilli, S., Homer, K. A., Yuste, J., Basavanna, S., Mitchell, T., and Brown, J. S. (2008). Maturation of Streptococcus pneumoniae lipoproteins by a type II signal peptidase is required for $\mathrm{ABC}$ transporter function and full virulence. Mol. Microbiol. 67, 541-557. doi: 10.1111/j.1365-2958.2007.06065.x

Kouwen, T. R., van der Goot, A., Dorenbos, R., Winter, T., Antelmann, H., Plaisier, M. C., et al. (2007). Thiol-disulphide oxidoreductase modules in the lowGC Gram-positive bacteria. Mol. Microbiol. 64, 984-999. doi: 10.1111/j.13652958.2007.05707.x

Kurokawa, K., Kim, M. S., Ichikawa, R., Ryu, K. H., Dohmae, N., Nakayama, H., et al. (2012). Environment-mediated accumulation of diacyl lipoproteins over their triacyl counterparts in Staphylococcus aureus. J. Bacteriol. 194, 3299-3306. doi: 10.1128/JB.00314-12

Kurokawa, K., Lee, H., Roh, K. B., Asanuma, M., Kim, Y. S., Nakyama, H., et al. (2009). The triacylated ATP binding cluster transporter substrate-binding lipoprotein of Staphylococcus aureus functions as a native ligand for the toll-like receptor 2. J. Biol. Chem. 284, 8406-8411. doi: 10.1074/jbc.M809618200

Machata, S., Tchatalbachev, S., Mohamed, W., Jänsch, L., Hain, T., and Chakraborty, T. (2008). Lipoproteins of Listeria monocytogenes are critical for virulence and TLR2-mediated immune activation. J. Immunol. 181, 2028-2035. doi: 10.4049/jimmunol.181.3.2028

Magadum, S., Banerjee, U., Murugan, P., Gangapur, D., and Ravikesavan, R. (2013). Gene duplication as a major force in evolution. J. Genet. 92, 155-161. doi: 10.1007/s12041-013-0212-8

Makgotlho, P. E., Marincola, G., Schäfer, D., Liu, Q., Bae, T., Geiger, T., et al. (2013). SDS interferes with SaeS signaling of Staphylococcus aureus independently of SaePQ. PLoS ONE 8:e71644. doi: 10.1371/journal.pone.0071644

Mariotti, P., Malito, E., Biancucci, M., Lo Surdo, P., Mishra, R. P., Nardi-Dei, V., et al. (2013). Structural and functional characterization of the Staphylococcus aureus virulence factor and vaccine candidate FhuD2. Biochem. J. 449, 683-693. doi: 10.1042/BJ20121426

Mazmanian, S. K., Skaar, E. P., Gaspar, A. H., Humayun, M., Gornicki, P., Jelenska, J., et al. (2003). Passage of heme-iron across the envelope of Staphylococcus aureus. Science 299, 906-909. doi: 10.1126/science.1081147

Mazmanian, S. K., Ton-That, H., and Schneewind, O. (2001). Sortase-catalysed anchoring of surface proteins to the cell wall of Staphylococcus aureus. Mol. Microbiol. 40, 1049-1057. doi: 10.1046/j.1365-2958.2001.02411.x

Mazmanian, S. K., Ton-That, H., Su, K., and Schneewind, O. (2002). An ironregulated sortase anchors a class of surface protein during Staphylococcus aureus pathogenesis. Proc. Natl. Acad. Sci. U.S.A. 99, 2293-2298. doi: 10.1073/pnas.032523999

Merlin, C., Gardiner, G., Durand, S., and Masters, M. (2002). The Escherichia coli metD locus encodes an $\mathrm{ABC}$ transporter which includes $\mathrm{Abc}(\mathrm{MetN})$, YaeE (MetI), and YaeC (MetQ). J. Bacteriol. 184, 5513-5517. doi: 10.1128/JB.184.19.5513-5517.2002

Morrissey, J. A., Cockayne, A., Hill, P. J., and Williams, P. (2000). Molecular cloning and analysis of a putative siderophore $\mathrm{ABC}$ transporter 
from Staphylococcus aureus. Infect. Immun. 68, 6281-6288. doi: 10.1128/IAI.68.11.6281-6288.2000

Müller, P., Müller-Anstett, M., Wagener, J., Gao, Q., Kaesler, S., Schaller, M., et al. (2010). The Staphylococcus aureus lipoprotein SitC colocalizes with Tolllike receptor 2 (TLR2) in murine keratinocytes and elicits intracellular TLR2 accumulation. Infect. Immun. 78, 4243-4250. doi: 10.1128/IAI.00538-10

Neubauer, H., Pantel, I., Lindgren, P. E., and Götz, F. (1999). Characterization of the molybdate transport system ModABC of Staphylococcus carnosus. Arch. Microbiol. 172, 109-115.

Nguyen, M. T., and Götz, F. (2016). Lipoproteins of Gram-Positive bacteria: key players in the immune response and virulence. Microbiol. Mol. Biol. Rev. 80, 891-903. doi: 10.1128/MMBR.00028-16

Nguyen, M. T., Hanzelmann, D., Hartner, T., Peschel, A., and Götz, F. (2015a). Skin-specific unsaturated fatty acids boost the Staphylococcus aureus innate immune response. Infect. Immun. 84, 205-215. doi: 10.1128/IAI.00822-15

Nguyen, M. T., Kraft, B., Yu, W., Demicrioglu, D. D., Hertlein, T., Burian, M., et al. (2015b). The $\nu$ Sa $\alpha$ specific Lipoprotein Like Cluster (lpl) of S. aureus USA300 contributes to immune stimulation and invasion in human cells. PLoS Pathog. 11:e1004984. doi: 10.1371/journal.ppat.1004984

Nielsen, J. B., and Lampen, J. O. (1982a). Glyceride-cysteine lipoproteins and secretion by Gram-positive bacteria. J. Bacteriol. 152, 315-322.

Nielsen, J. B., and Lampen, J. O. (1982b). Membrane-bound penicillinases in Gram-positive bacteria. J. Biol. Chem. 257, 4490-4495.

Petit, C. M., Brown, J. R., Ingraham, K., Bryant, A. P., and Holmes, D. J. (2001). Lipid modification of prelipoproteins is dispensable for growth in vitro but essential for virulence in Streptococcus pneumoniae. FEMS Microbiol. Lett. 200, 229-233. doi: 10.1111/j.1574-6968.2001.tb10720.x

Podkowa, K. J., Briere, L. A., Heinrichs, D. E., and Shilton, B. H. (2014). Crystal and solution structure analysis of FhuD2 from Staphylococcus aureus in multiple unliganded conformations and bound to ferrioxamine-B. Biochemistry 53, 2017-2031. doi: 10.1021/bi401349d

Rahman, O., Cummings, S. P., Harrington, D. J., and Sutcliffe, I. C. (2008). Methods for the bioinformatic identification of bacterial lipoproteins encoded in the genomes of Gram-positive bacteria. World J. Microbiol. Biotechnol. 24, 2377-2382. doi: 10.1007/s11274-008-9795-2

Remy, L., Carrière, M., Derré-Bobillot, A., Martini, C., Sanguinetti, M., and Borezee-Durant, E. (2013). The Staphylococcus aureus Opp1 ABC transporter imports nickel and cobalt in zinc-depleted conditions and contributes to virulence. Mol. Microbiol. 87, 730-743. doi: 10.1111/mmi.12126

Robinson, D. A., Kearns, A. M., Holmes, A., Morrison, D., Grundmann, H., Edwards, G., et al. (2005). Re-emergence of early pandemic Staphylococcus aureus as a community-acquired meticillin-resistant clone. Lancet 365, 1256-1258. doi: 10.1016/S0140-673674814-5

Sander, P., Rezwan, M., Walker, B., Rampini, S. K., Kroppenstedt, R. M., Ehlers, S., et al. (2004). Lipoprotein processing is required for virulence of Mycobacterium tuberculosis. Mol. Microbiol. 52, 1543-1552. doi: 10.1111/j.1365-2958.2004.04041.x

Sankaran, K., and Wu, H. C. (1994). Lipid modification of bacterial prolipoprotein. Transfer of diacylglyceryl moiety from phosphatidylglycerol. J. Biol. Chem. 269, 19701-19706.

Schluepen, C., Malito, E., Marongiu, A., Schirle, M., McWhinnie, E., Lo Surdo, P., et al. (2013). Mining the bacterial unknown proteome: identification and characterization of a novel family of highly conserved protective antigens in Staphylococcus aureus. Biochem. J. 455, 273-284. doi: 10.1042/BJ20130540

Schmaler, M., Jann, N. J., Ferracin, F., Landolt, L. Z., Biswas, L., Götz, F., et al. (2009). Lipoproteins in Staphylococcus aureus mediate inflammation by TLR2 and iron-dependent growth in vivo. J. Immunol. 182, 7110-7118. doi: 10.4049/jimmunol.0804292

Schmaler, M., Jann, N. J., Götz, F., and Landmann, R. (2010). Staphylococcal lipoproteins and their role in bacterial survival in mice. Int. J. Med. Microbiol. 300, 155-160. doi: 10.1016/j.ijmm.2009.08.018

Schmollinger, M., Fischer, I., Nerz, C., Pinkenburg, S., Götz, F., Kaufmann, M., et al. (2004). ParSeq: searching motifs with structural and biochemical properties. Bioinformatics 20, 1459-1461. doi: 10.1093/bioinformatics/bth083

Sebulsky, M. T., and Heinrichs, D. E. (2001). Identification and characterization of fhuD1 and fhuD2, two genes involved in iron-hydroxamate uptake in Staphylococcus aureus. J. Bacteriol. 183, 4994-5000. doi: 10.1128/JB.183.17.4994-5000.2001
Sebulsky, M. T., Hohnstein, D., Hunter, M. D., and Heinrichs, D. E. (2000) Identification and characterization of a membrane permease involved in ironhydroxamate transport in Staphylococcus aureus. J. Bacteriol. 182, 4394-4400. doi: 10.1128/JB.182.16.4394-4400.2000

Sebulsky, M. T., Speziali, C. D., Shilton, B. H., Edgell, D. R., and Heinrichs, D. E. (2004). FhuD1, a ferric hydroxamate-binding lipoprotein in Staphylococcus aureus: a case of gene duplication and lateral transfer. J. Biol. Chem. 279, 53152-53159. doi: 10.1074/jbc.M409793200

Sibbald, M. J., Ziebandt, A. K., Engelmann, S., Hecker, M., de Jong, A., Harmsen, H. J., et al. (2006). Mapping the pathways to staphylococcal pathogenesis by comparative secretomics. Microbiol. Mol. Biol. Rev. 70, 755-788. doi: 10.1128/MMBR.00008-06

Skabytska, Y., Wölbing, F., Gunther, C., Koberle, M., Kaesler, S., Chen, K. M., et al. (2014). Cutaneous innate immune sensing of Toll-like receptor 2-6 ligands suppresses $\mathrm{T}$ cell immunity by inducing myeloid-derived suppressor cells. Immunity 41, 762-775. doi: 10.1016/j.immuni.2014.10.009

Stoll, H., Dengjel, J., Nerz, C., and Götz, F. (2005). Staphylococcus aureus deficient in lipidation of prelipoproteins is attenuated in growth and immune activation. Infect. Immun. 73, 2411-2423. doi: 10.1128/IAI.73.4.2411-2423.2005

Sutcliffe, I. C., and Harrington, D. J. (2002). Pattern searches for the identification of putative lipoprotein genes in Gram-positive bacterial genomes. Microbiology 148(Pt 7), 2065-2077. doi: 10.1099/00221287-148-7-2065

Sutcliffe, I. C., Harrington, D. J., and Hutchings, M. I. (2012). A phylum level analysis reveals lipoprotein biosynthesis to be a fundamental property of bacteria. Protein Cell 3, 163-170. doi: 10.1007/s13238-012-2023-8

Takeda, K., Takeuchi, O., and Akira, S. (2002). Recognition of lipopeptides by Toll-like receptors. J. Endotoxin Res. 8, 459-463. doi: 10.1179/0968051021250 01073

Takeuchi, O., Kawai, T., Mühlradt, P. F., Morr, M., Radolf, J. D., Zychlinsky, A., et al. (2001). Discrimination of bacterial lipoproteins by Toll-like receptor 6 . Int. Immunol. 13, 933-940. doi: 10.1093/intimm/13.7.933

Takeuchi, O., Sato, S., Horiuchi, T., Hoshino, K., Takeda, K., Dong, Z., et al. (2002). Cutting edge: role of Toll-like receptor 1 in mediating immune response to microbial lipoproteins. J. Immunol. 169, 10-14. doi: 10.4049/jimmunol.169.1.10

Taylor, P. D., Toseland, C. P., Attwood, T. K., and Flower, D. R. (2006). LIPPRED: A web server for accurate prediction of lipoprotein signal sequences and cleavage sites. Bioinformation 1, 176-179. doi: 10.6026/97320630001176

von Heijne, G. (1989). The structure of signal peptides from bacterial lipoproteins. Protein Eng. 2, 531-534.

Vu, C. H., Kolata, J., Stentzel, S., Beyer, A., Salazar, M. G., Steil, L., et al. (2016) Adaptive immune response to lipoproteins of Staphylococcus aureus in healthy subjects. Proteomics. doi: 10.1002/pmic.201600151. [Epub ahead of print].

Wang, P., and Dalbey, R. E. (2011). Inserting membrane proteins: the YidC/Oxa1/Alb3 machinery in bacteria, mitochondria, and chloroplasts. Biochim. Biophys. Acta 1808, 866-875. doi: 10.1016/j.bbamem.2010.08.014

Weston, B. F., Brenot, A., and Caparon, M. G. (2009). The metal homeostasis protein, Lsp, of Streptococcus pyogenes is necessary for acquisition of zinc and virulence. Infect. Immun. 77, 2840-2848. doi: 10.1128/IAI.01299-08

Williams, W. A., Zhang, R. G., Zhou, M., Joachimiak, G., Gornicki, P., Missiakas, D., et al. (2004). The membrane-associated lipoprotein-9 GmpC from Staphylococcus aureus binds the dipeptide GlyMet via side chain interactions. Biochemistry 43, 16193-16202. doi: 10.1021/bi048877o

Zähringer, U., Lindner, B., Inamura, S., Heine, H., and Alexander, C. (2008). TLR2 - promiscuous or specific? A critical re-evaluation of a receptor expressing apparent broad specificity. Immunobiology 213, 205-224. doi: 10.1016/j.imbio.2008.02.005

Conflict of Interest Statement: The authors declare that the research was conducted in the absence of any commercial or financial relationships that could be construed as a potential conflict of interest.

Copyright (c) 2016 Shahmirzadi, Nguyen and Götz. This is an open-access article distributed under the terms of the Creative Commons Attribution License (CC BY). The use, distribution or reproduction in other forums is permitted, provided the original author(s) or licensor are credited and that the original publication in this journal is cited, in accordance with accepted academic practice. No use, distribution or reproduction is permitted which does not comply with these terms. 